This document was prepared in conjunction with work accomplished under Contract No. DE-AC09-96SR18500 with the U. S. Department of Energy.

\title{
DISCLAIMER
}

This report was prepared as an account of work sponsored by an agency of the United States Government. Neither the United States Government nor any agency thereof, nor any of their employees, nor any of their contractors, subcontractors or their employees, makes any warranty, express or implied, or assumes any legal liability or responsibility for the accuracy, completeness, or any third party's use or the results of such use of any information, apparatus, product, or process disclosed, or represents that its use would not infringe privately owned rights. Reference herein to any specific commercial product, process, or service by trade name, trademark, manufacturer, or otherwise, does not necessarily constitute or imply its endorsement, recommendation, or favoring by the United States Government or any agency thereof or its contractors or subcontractors. The views and opinions of authors expressed herein do not necessarily state or reflect those of the United States Government or any agency thereof. 
WSRC-RP-2005-01405

\section{Savannah River Site Annual Meteorology Report for 2004 (U)}

\section{H. H unter}

\section{Westinghouse Savannah River Company}

Savannah River Site

Aiken, SC 29808

Prepared for the U. S. Department of Energy under contract no. DE-AC09-96SR18500 
This page was intentionally left blank 


\section{Contents}

$\begin{array}{ll}\text { Summary } & 1\end{array}$

$\begin{array}{lr}\text { Background } & 2\end{array}$

The General SRS Climate 2

Overview of Meteorological Monitoring at the 2

Savannah River Site

Data Sources for the 2004 Report 3

The SRS Climatology for 2004

Highlights $\quad 5$

Temperature $\quad 5$

Precipitation 6

Atmospheric Moisture 6

Heat Stress $\quad 6$

Wind $\quad 7$

Barometric Pressure $\quad 7$

$\begin{array}{ll}\text { Solar Radiation } & 7\end{array}$

$\begin{array}{lr}\text { References } & 9\end{array}$

$\begin{array}{ll}\text { Appendix A } & 32\end{array}$ 


\section{List of Tables}

Table 1. Means and Extremes for 2004

(a) Temperature and Precipitation

(b) Dew Point, Wet Bulb, Relative Humidity, and Wet Bulb Globe Temperature

(c) Wind Speed, Barometric Pressure, Solar Radiation, and Heating / Cooling Degree Days

Table 2. Monthly and Annual Average and Extreme Temperatures, 1974-2004

Table 3. Monthly and Annual Average and Extreme Rainfall, 1974-2004

Table 4. Monthly and Annual Rainfall, Manual Gauges

Table A Joint Occurrence Frequencies of Wind Direction Sector by Wind Speed Wind Speed Category, 2004
A.1 A Area Tower
A.2 C Area Tower
A.3 D Area Tower
A.4 F Area Tower
A.5 H Area Tower
A.6 K Area Tower
A.7 L Area Tower
A.8 P Area Tower
A.9 2m Level Central Climatology Tower
A.10 18m Level Central Climatology Tower
A.11 36m Level Central Climatology Tower
A.12 61m Level Central Climatology Tower
A.13 61m Level Central Climatology Tower, Winter
A.14 61m Level Central Climatology Tower, Spring
A.15 61m Level Central Climatology Tower, Summer
A.16 61m Level Central Climatology Tower, Fall 


\section{List of Figures}

Figure 1. Meteorological Monitoring Stations

Figure 2. Summary of Daily Data for 2004

Figure 3. Daily High and Low Temperatures for 2004

Figure 4. SRS Annual Average Temperature 1973-2004

Figure 5. SRS Monthly Average Temperature

Figure 6. Number of Freezing ( $<32 \mathrm{~F})$ and Sweltering $(>90 \mathrm{~F})$ Days

Figure 7. Daily Precipitation for 2004

Figure 8. SRS Annual Precipitation 1973-2004

Figure 9. SRS Monthly Precipitation

Figure 10. Daily High and Low Humidity for 2004

Figure 11. SRS Annual Average Humidity 1973-2004

Figure 12. SRS Monthly Average Minimum Humidity

Figure 13. Daily Average Wind Speed for 2004

Figure 14. Annual Wind Rose Plots for 2004, 61m Level

(a) A, C, D, and F Area Towers

(b) H, K, L, and P Area Towers

Figure 15. Annual Wind Rose Plots for 2004, Central Climatology Tower, All Levels

Figure 16. Seasonal Wind Rose Plots for 2004, Central Climatology Tower, 61m Level

Figure 17. Daily Average Barometric Pressure for 2004

Figure 18. Daily Total Solar Radiation for 2004 


\section{Summary}

Summaries of meteorological observations collected at the Savannah River Site in 2004 show a year that was overall cooler and drier than average. Although the annual rainfall of 42.9 inches was the eleventh driest of all the years over a period of record that began in 1952, rainfall was quite variable through the year. September's total rainfall of 10.26 inches was the highest in this 53 year record; conversely, the monthly rainfall in March, 0.81 inches, was the lowest on record. Rainfall of 0.01 inch or more occurred on 104 days during the year.

The annual average temperature for 2004, $63.4 \mathrm{oF}$, was the eleventh coldest of any year in an available record that dates to 1964. Cooler than average conditions were observed in 9 of the 12 months of the year. The coldest temperature during the year was $20.3 \circ \mathrm{F}$ on the morning of December 15; the warmest observed temperature was $98.2 \mathrm{~F}$ on the afternoon of July 14 .
The most notable weather event of 2004 was an active Atlantic hurricane season that resulted in six named storms striking the Southeast U.S. during August and September. Although each of these storms posed a significant threat to the SRS, their eventual paths resulted in only minimal impacts. The remnants of hurricanes Frances and Jeanne produced 24-hr rainfall totals of 3.99 inches (Sept. 8) and 3.48 inches (Sept. 27), respectively. Surface winds associated with Jeanne resulted in sustained speeds approaching $15 \mathrm{mph}$ with gusts to $35 \mathrm{mph}$ on September 27.

An ice storm on January 26 produced an estimated accumulation of one-fourth to one-half inch of ice, resulting in scattered power outages and considerable damage to trees across the Site.

A strong cold front moving through the area on March 7 produced a 15-minute average surface wind of $24 \mathrm{mph}$ with a gust to near $50 \mathrm{mph}$. 


\section{Background}

\section{The General SRS Climate}

The Savannah River Site region has a humid subtropical climate characterized by relatively short, mild winters and long, warm, and humid summers (Oliver and Fairbridge, 1987).

Summer-like conditions typically last from May through September, when the area is frequently under the influence of the western extension of the semi-permanent Atlantic subtropical anticyclone (i.e. the 'Bermuda' high). Winds in summer are light and cold fronts generally remain well north of the area. Daily high temperatures during the summer months exceed $90^{\circ} \mathrm{F}$ on more than half of all days on the average. Scattered afternoon and evening thunderstorms are common.

The influence of the Bermuda high begins to diminish during the fall, resulting in lower humidity and more moderate temperatures. Average rainfall during the fall is usually the least of the four seasons.

In the winter months, mid-latitude low pressure systems and associated fronts often migrate through the region. As a result, conditions frequently alternate between warm, moist, subtropical air from the Gulf of Mexico region and cool, dry polar air. The Appalachian Mountains to the north and northwest of the SRS help to moderate the extremely cold temperatures associated with occasional outbreaks of Arctic air. Consequently, less than onethird of winter days have minimum temperatures below freezing on average, and days with temperatures below $20^{\circ} \mathrm{F}$ are infrequent. Measurable snowfall occurs an average of once every 1-2 years.

Tornadoes occur more frequently in spring than the other seasons of the year. Although spring weather is somewhat windy, temperatures are usually mild and humidity is relatively low.

\section{Overview of the Savannah River Site Meteorological Monitoring Program}

Meteorological data are collected at SRS from a network of nine primary monitoring stations (Fig. 1). Towers located adjacent to each of eight primary operations areas (A, C, D, F, H, K, L, and $\mathrm{P}$ areas) are equipped to measure wind direction, wind speed, temperature, and dew point at a height of 61 meters (m) above ground. Temperature and dew point are also measured at $2 \mathrm{~m}$. A ninth tower near $\mathrm{N}$ Area, known as the Central Climatology site (CLM), is instrumented with wind, temperature, and dew point sensors at four levels: $2 \mathrm{~m}$ ( $4 \mathrm{~m}$ for wind), $18 \mathrm{~m}, 36 \mathrm{~m}$, and $61 \mathrm{~m}$. The CLM site is also equipped with an automated tipping bucket rain gauge, a barometric pressure sensor, and a solar radiometer near the tower at ground level. Data acquisition units at each station record a measurement from each instrument at 1second intervals. Every 15 minutes, the 1second data are processed to generate statistical summaries for each variable, including averages and instantaneous maxima, and the results are uploaded to a relational database for permanent archival. All aspects of the meteorological data collection program meet or exceed applicable regulatory criteria. Parker and Addis (1993) provide a complete description of the meteorological monitoring program at SRS.

Quality assurance of the data is conducted in two phases: an initial screening of recent 
data, followed by an in-depth review and final quality classification. The initial screening, performed twice daily by qualified instrument technicians, consists of a thorough examination of 15-minute data retrieved from the database, in conjunction with a summary of instrument diagnostics obtained from the local data acquisition units. Potential problems are noted in a daily checksheet and, as needed, data acquisition unit software is instructed to assign a quality control tag to data collected from questionable instruments. Quality tags are also set during periods of calibration and maintenance.

The second phase of the quality assurance process is conducted according to formal procedure (SRNL, 2004). Daily checksheets generated during the initial screening, tower-specific logbook entries, initial quality tags, and time series plots of related data are reviewed to determine a final quality status for each record. All records permanently archived in the data base are identified as good, fatal, intermittent, biased, or uncalibrated.

\section{Additional Measurements}

Additional precipitation measurements are collected from a network of 12 plastic wedge rain gauges across the SRS (See Fig. 1). These gauges are read manually by security or operations personnel once per day, usually around $6 \mathrm{am}$. The daily data are reported to the SRS Atmospheric Technologies Center, reviewed to correct obvious flaws, and manually entered into a permanent electronic data base.

Additional measurements of temperature and relative humidity are recorded from a station located in A-Area, adjacent to 773A. This station consists of a standard National Weather Service 'cotton region' instrument shelter. Data collected from this station are manually tabulated for archival as daily high and low values of temperature and relative humidity. Tabulated values are then entered into a permanent electronic data base.

A major enhancement to the SRS meteorological monitoring program during 2004 was the resumption of data collection from instrumentation on a nearby television transmission tower. The new system utilizes fast-response sonic anemometers, water vapor sensors, and barometric pressure sensors, and slow-response temperature sensors and relative humidity sensors. Data are collected at $30 \mathrm{~m}, 61 \mathrm{~m}$, and $304 \mathrm{~m}$ above ground level. Spreadspectrum modems at each measurement level transmit raw data to a redundant set of PCs at SRNL. Data processing software on the PCs determine mean values and other statistical quantities every 15 minutes and uploads the results to the relational database. The detailed data sets produced by this system are expected to provide a valuable resource for use in ATG's operational weather forecasting activities, emergency response, atmospheric boundary layer research, and climate change studies.

\section{Data Sources for the 2004 Report}

Data summaries provided with this report consist of the following:

- Ground level measurements of temperature (2m), dew point temperature (2m), wind speed $(4 m)$, precipitation, barometric pressure, and solar radiation from CLM, as well as meteorological quantities derived from these primary data, such as relative humidity (temperature and dew point), wet bulb temperature (temperature and dew point), wet bulb globe temperature 
(temperature, dew point, wind speed, and solar radiation), and cooling/heating degree days (temperature).

- Joint occurrence frequencies of wind speed and direction from measurements taken at each of the four levels of the CLM tower and the $61 \mathrm{~m}$ level of the eight area towers.

- Precipitation from the network of manually-read rain gauges

A series of SAS System software routines were used to extract the desired data sets from the data base and perform initial statistical processing on all records with a QA status of 'good'. More than $99 \%$ of the 2004 data used in this report met this criterion with one exception. The 2 meter dew point measurement was flagged as unacceptable from the period May 21 through August 16. Dew point data from the CLM 18-meter level was used as a substitute data source.

Output from the initial processing was then imported into an Excel spreadsheet to generate the final summaries that are presented in the report.

Long-term climatological records are available for temperature, precipitation, and relative humidity. Statistics generated from these data are used for comparisons with the 2004 summaries.

Available temperature and relative humidity records begin in 1964 . From 1964-1995, climatological statistics used in this report were based on the record of daily high and low values recorded at the 773-A instrument shelter. After 1995, these statistics were based on the continuous record of 15-minute data from the $2 \mathrm{~m}$ level of CLM.
Precipitation data are available from 1952. For the period 1952 through 1995, climatological statistics used in the report were based on the daily observations from the 773-A rain gauge. Summaries after 1995 were based on the automated 15minute records collected from the CLM rain gauge. 


\section{Climatology of the SRS for 2004}

\section{Highlights}

Meteorological data summaries for 2004 indicate that overall weather conditions at SRS were slightly cooler and drier than long-term averages. The total annual precipitation of 42.9 inches was the eleventh driest of all years in the available period of record. The annual average temperature of $63.4^{\circ} \mathrm{F}$ was the eleventh coldest of any year in the available record. Cooler than average conditions were observed throughout the summer months; otherwise, there were no prolonged departures from long-term average temperatures. Dry conditions during most of spring and summer period was offset by record rainfall in September. A summary plot of daily observations of temperature, precipitation, relative humidity, and wind speed is given in Fig. 2.

Monthly climatological discussions published by the National Weather Service, Climate Prediction Center (CPC), indicate that the relatively cool weather observed throughout much of the eastern U. S. for June through August was due to the persistent presence of a low pressure trough across east-central Canada from Hudson Bay southward to the Great Lakes region, and a relatively weak presence of a subtropical (Bermuda) high over the southwest Atlantic and Southeastern U.S. West to northwest flow that developed on the southwestern flank of this trough enabled cool fronts to propagate through the Southeast on several occasions.

During the latter half of August through September, the Bermuda high strengthened and centered over a position just off the U.S. east coast. The clockwise circulation around the periphery of this high provided the mechanism for steering tropical systems westward then northward into Florida and Southeast U.S.

The upper trough returned to a position along the east coast on several occasions in December, accompanied by a strong polar jet that was responsible for several outbreaks of cold arctic air from Canada (NOAA, 2004a and 2004b).

\section{Temperature}

Monthly and annual average and daily extreme temperatures for 2004 are summarized in Table 1(a). Similar statistics for a 30-year climatological reference period (1974-2003) are given in Table 2. A plot of observed daily high and low temperatures for 2004 is shown in Fig. 3. Plots of annual average temperature, monthly average daily high and low temperature, and days exceeding critical temperature thresholds $(<32 \mathrm{\circ}$, $>90 \circ \mathrm{F})$ for 2004 and the 30-year reference period are shown in figures 4,5 , and 6 , respectively.

The annual average temperature of $63.4 \mathrm{oF}$ was 1.2 degrees below the 30-year average. Monthly averages for 2004 were below their respective climatological values for all months in the year except March, May, and October. Furthermore, monthly averages for February and August ranked among the five coldest months on record. Temperatures above $90^{\circ} \mathrm{F}$ occurred on a total of 52 days during the year and only one-half of total days in July and August. Temperature extremes ranged from $20.3 \mathrm{oF}$ on December 15 to 98.2 F on July 14. Daily low temperature records were set on February 29, April 15, August 7, and December 15 (tie) and 16. Daily high temperature records were set on January 5 and March 28. 
Monthly and annual total heating and cooling degree days (based on a reference temperature of $65^{\circ} \mathrm{F}$ ) are summarized in Table 1(c).

\section{Precipitation}

Annual, monthly, and daily total rainfall statistics for 2004 are summarized in Table 1(a). Monthly and annual rainfall totals for the previous 30-years (1974-2003) are given in Table 3. Monthly and annual rainfall totals for the 12 plastic wedge gauges are summarized in Table 4. A plot of daily total rainfall (midnight to midnight) for the CLM site for 2004 is shown as Fig. 7. Annual and monthly rainfall for 2004 and the 30-year reference period are shown in figures 8 and 9 , respectively.

Total precipitation at the CLM site, 42.9 inches, was about six inches less than the 30 -year average and resulted in the eleventh driest year over the 53 years of available data. Monthly rainfall for March and July were the lowest and second lowest totals on record for those months, respectively. Conversely, September's total of 10.26 inches was the highest on record for the month. The heavy rain that occurred on September 8 (3.99 in) was due to the remnants of Hurricane Jeanne.

Measurable precipitation ( $>0.01$ inch) occurred on 104 days and rainfall greater than 0.5 inch occurred on 23 days.

\section{Atmospheric Moisture}

Monthly and annual average and daily extreme dew point temperature, wet bulb temperature, and relative humidity for 2004 are summarized in Table 1(b). This table also presents monthly and annual average daily maximum and minimum humidity. A plot of daily maximum and minimum humidity for 2004 is shown in Fig. 10. Plots of annual average humidity for 2004 and the 30-year reference period (1974-2003) and monthly average daily minimum humidity are shown in figures 11 and 12, respectively.

Average relative humidity for 2004 was 68 percent (\%) with an average daily minimum of $45 \%$ and an average daily maximum of $86 \%$. Days with relative humidity of $20 \%$ or less occurred in January, February, March, April, and December. The lowest relative humidity recorded during the year was $16 \%$ on April 4. Average daily minimum relative humidity was lower than long-term averages in March and April and greater than long-term averages during the fall.

Observed dew point temperatures ranged from $77.4 \mathrm{oF}$ on September 7 to $5.1 \mathrm{oF}$ on December 20. Wet bulb temperature ranged from $78.1 \mathrm{oF}$ on September 1 to $18.3 \mathrm{oF}$ on December 20.

\section{Heat Stress}

Restrictions on outdoor work due to excessive heat are based on values of the wet bulb globe temperature (WBGT) (WSRC, 1995). Hunter and Minyard (2000) provide a description of WBGT and the method used at SRS to calculate WBGT from standard meteorological measurements at CLM.

Monthly and annual maximum WBGT and statistics on heat stress category days for 2004 is summarized in Table 1(b). The highest WBGT for 2004 was $91.5 \mathrm{oF}$ on July 12. Heat stress Category 5 (WBGT $>90.0$ $\circ \mathrm{F})$ occurred on 11 days during the summer of 2004. A total of 167 days reached at least 
Category 1 (WBGT > 77.0 oF) from March through November of the year.

\section{Wind}

Monthly average and extreme wind speed at the CLM 4 meter level for 2004 is summarized in Table 1(c). Daily averaged wind speed for the year is plotted in Fig. 13. Figures 14(a) and 14(b) show wind rose plots depicting joint occurrence frequencies of the indicated wind speed category by wind direction sector at the 61 meter level of the eight area towers. Fig. 15 provides wind rose plots for the 4 levels of measurement at CLM. Seasonal wind rose plots for 2004 for data from the 61m level of CLM are shown in Fig. 16. Tables of the joint frequency data used to create the wind rose plots are given in Appendix A.

Wind speeds generally were strongest on average during the winter and spring months. The highest instantaneous wind speed recorded at the $4 \mathrm{~m}$ level, $49.9 \mathrm{mph}$, occurred during the passage of a cold front on the evening of March 7. A strong winter storm moving through the Southeast U. S. on February 7 produced the highest daily average wind speed of 10.6 $\mathrm{mph}$. The highest monthly average wind speed occurred in September due to the unusually high number of tropical weather systems affecting the area.

Wind rose plots for the area towers show typical annual patterns for the 61 meter level. This pattern consists of higher frequencies of wind from the northeasterly sectors and southwest to westerly sectors. Due to the location of the D area tower in the shallow valley formed by the Savannah River, wind direction is more frequently from the southeasterly and northwesterly sectors than for the other area towers. Wind roses for CLM also show typical variations in the frequency patterns by level, with progressively higher frequencies of southeasterly winds and lower frequencies of northeasterly and southwesterly winds nearer the ground. Weber (2003) provides a complete description of the wind climatology at the CLM site.

Seasonal wind rose plots for the 61 meter level at CLM are indicative of the typical seasonal patterns observed at SRS.

\section{Barometric Pressure}

Annual and monthly average and extreme barometric pressure is summarized in Table 1(c). Daily average barometric pressure is plotted in Fig. 17. The lowest daily average and 15-minute minimum barometric pressure, 992.1 and $990.3 \mathrm{mb}$, respectively, were associated with the passage of a cold front on October 15. The highest daily average and 15-minute maximum barometric pressure, 1020.1 and $1027.2 \mathrm{mb}$, respectively, were associated with strong polar high pressure that built over the Southeast U. S. November 14 and 15.

\section{Solar Radiation}

Annual and monthly averages and extremes of daily total solar radiation are summarized in Table 1(c). This table also provides estimated monthly and annual estimates of percent observed solar radiation relative to theoretical clear sky maxima as an indicator of relative cloudiness. The monthly theoretical values were estimated from tables published by Budyko (1974). Daily total solar radiation for 2004 is plotted in Fig. 18. The average daily values ranged from 242 langleys per day (ly/day) in December to 544 ly/day in 
May. The low value for daily average solar radiation in December is due to a low solar angle, as the percent of theoretical maximum for the month, $73 \%$, implies December was characterized by ample sunshine. Sunny days were also prevalent March and April. Individual daily extremes ranged from $28 \mathrm{ly} /$ day on January 25 to 712 ly/day on July 8 . 


\section{References}

Budyko, M. I., Climate and Life, Academic Press, New York, NY (1974).

Hunter, C. H., and C. O. Minyard, Estimating Wet Bulb Globe Temperature Using Standard Meteorological Measurements, American Meteorological Society $2^{\text {nd }}$ Symposium on Environmental Applications, January 9-14, 2000, Long Beach, CA (2000).

National Oceanic and Atmospheric Administration (NOAA), Climate Diagnostics Bulletin (January - December 2004), published electronically at www.cpc.ncep.noaa.gov, Climate Prediction Center, Camp Springs, MD(2004a).

NOAA, Daily Weather Maps, National Weather Service, Camp Springs, MD.(2004b)

Oliver, J. E. and R. W. Fairbridge, ed., The Encyclopedia of Climatology, Von Nostrand Reinhold, New York, NY (1987).

Parker, M. J. and R. P. Addis, Meteorological Monitoring Program at the Savannah River Site, WSRC-TR-93-0106, Westinghouse Savannah River Company (1993).

SRNL Nonproliferation Technologies Section, Quality Assurance of Meteorological
Data, WSRC Procedure Manual 15.3, Meteorological Monitoring Procedures, NTSP T-113 (2004).

Weber, A. H., R. J. Kurzeja and R. L. Buckley, Wind Climate Analysis for SRTC's Central Climatology Site, WSRC-TR-200300141, Westinghouse Savannah River Company, Aiken, SC (2003).

Westinghouse Savannah River Company (WSRC), The SRS Heat Stress Management Program, Procedure Manual 4Q, Procedure 502, Revision 2 (1995). 
Table 1(a) - Means and Extremes of SRS Meteorological Data for 2004

\begin{tabular}{|c|c|c|c|c|c|c|c|c|c|c|c|c|c|c|c|c|c|c|c|c|c|}
\hline \multirow[b]{3}{*}{ 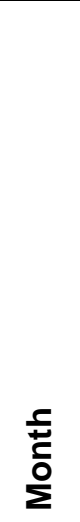 } & \multicolumn{13}{|c|}{ Temperatures $\left({ }^{\circ} \mathrm{F}\right)$} & \multicolumn{8}{|c|}{ Precipitation (in) } \\
\hline & \multicolumn{5}{|c|}{ Average } & \multicolumn{4}{|c|}{ Extremes } & \multicolumn{4}{|c|}{ Number of Days } & \multirow{2}{*}{\multicolumn{2}{|c|}{ 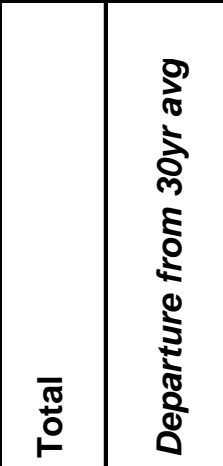 }} & \multirow[b]{2}{*}{ 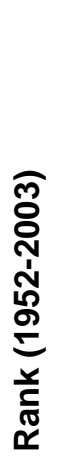 } & \multirow[b]{2}{*}{ 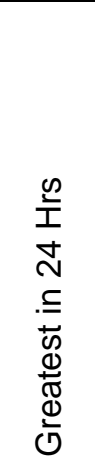 } & \multirow[b]{2}{*}{ 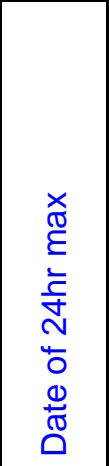 } & \multicolumn{3}{|c|}{ No. of Days } \\
\hline & 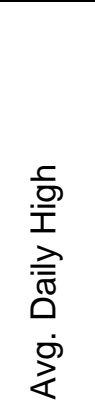 & 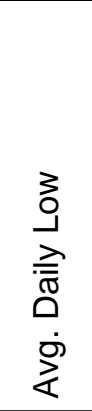 & 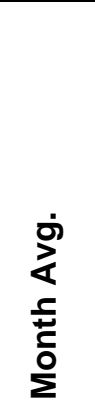 & 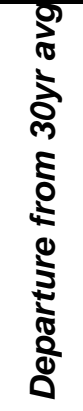 & 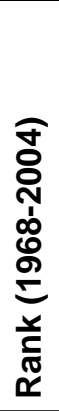 & 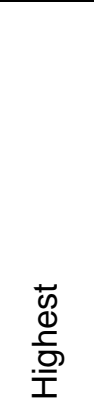 & 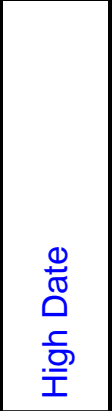 & 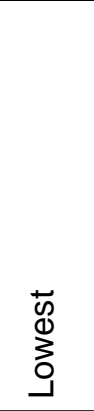 & 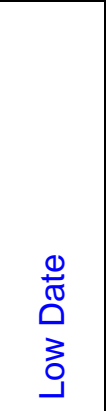 & 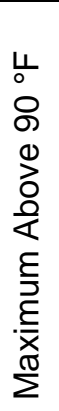 & 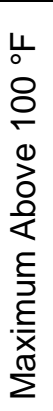 & 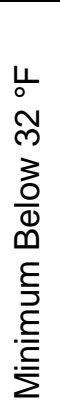 & 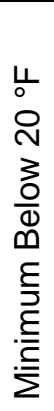 & & & & & & 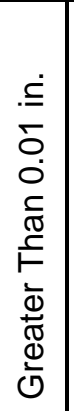 & 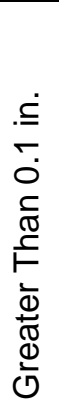 & 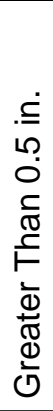 \\
\hline Jan & 56.8 & 33.5 & 43.7 & -2.3 & 13 & 75.9 & 5 th & 20.9 & 8th & 0 & 0 & 17 & 0 & 2.85 & -1.49 & 13 & 1.74 & 26th & 8 & 5 & 1 \\
\hline Feb & 55.8 & 36.7 & 45.2 & -4.8 & 5 & 72.3 & 6th & 25.2 & 8th & 0 & 0 & 7 & 0 & 6.71 & +2.29 & 9 & 2.01 & 26th & 11 & 8 & 5 \\
\hline Mar & 71.4 & 46.6 & 58.5 & +1.3 & 12 & 84.1 & 28th & 29.9 & 11th & 0 & 0 & 2 & 0 & 0.81 & -3.92 & 1 & 0.18 & $21 \mathrm{st}$ & 6 & 5 & 0 \\
\hline Apr & 77.4 & 49.7 & 63.4 & -0.9 & 12 & 88.8 & 24th & 33.1 & 5 th & 0 & 0 & 0 & 0 & 1.34 & -1.90 & 10 & 0.64 & 27th & 6 & 3 & 1 \\
\hline May & 86.1 & 63.4 & 74.0 & +1.9 & 11 & 93.7 & 26th & 44.3 & 4th & 12 & 0 & 0 & 0 & 3.45 & -0.15 & 26 & 1.64 & 12th & 10 & 4 & 2 \\
\hline Jun & 88.7 & 69.3 & 77.7 & -0.7 & 14 & 95.4 & 12th & 64.9 & $1 \mathrm{st}$ & 9 & 0 & 0 & 0 & 6.41 & +1.73 & 11 & 1.59 & 30th & 14 & 11 & 5 \\
\hline Jul & 91.7 & 71.1 & 80.1 & -1.4 & 10 & 98.2 & 14th & 66.2 & 20th & 22 & 0 & 0 & 0 & 1.23 & -4.23 & 2 & 0.52 & $3 r d$ & 9 & 3 & 1 \\
\hline Aug & 87.6 & 68.9 & 77.3 & -2.9 & 4 & 96.6 & 4th & 56.1 & 8th & 9 & 0 & 0 & 0 & 2.96 & -1.96 & 13 & 1.25 & $31 \mathrm{st}$ & 11 & 8 & 1 \\
\hline Sep & 81.7 & 66.6 & 73.2 & -1.9 & 9 & 88.4 & $1 \mathrm{st}$ & 54.2 & 20th & 0 & 0 & 0 & 0 & 10.26 & +6.05 & 1 & 3.99 & 8th & 7 & 6 & 4 \\
\hline Oct & 75.5 & 59.1 & 66.2 & +0.9 & 15 & 88.1 & 2nd & 42.4 & 16th & 0 & 0 & 0 & 0 & 1.02 & -2.07 & 16 & 0.40 & 15th & 10 & 2 & 0 \\
\hline Nov & 67.3 & 47.1 & 56.1 & -0.4 & 18 & 82.1 & $1 \mathrm{st}$ & 30.7 & 26th & 0 & 0 & 3 & 0 & 3.17 & +0.20 & 16 & 1.59 & 24th & 8 & 6 & 1 \\
\hline Dec & 58.8 & 35.8 & 45.8 & -2.4 & 11 & 76.6 & 9th & 20.3 & 15th & 0 & 0 & 12 & 0 & 2.69 & -0.61 & 20 & 1.29 & 10th & 4 & 3 & 2 \\
\hline Year & 75.0 & 54.0 & 63.4 & -1.2 & 11 & 98.2 & 14-Jul & 20.3 & 15-Dec & 52 & 0 & 41 & 0 & 42.90 & -6.06 & 11 & 3.99 & 8-Sep & 104 & 64 & 23 \\
\hline
\end{tabular}


Table 1(b) - Means and Extremes of SRS Meteorological Data for 2004

\begin{tabular}{|c|c|c|c|c|c|c|c|c|c|c|c|c|c|c|c|c|c|c|c|c|c|c|}
\hline \multirow[b]{3}{*}{$\begin{array}{l}\text { 들 } \\
\text { 들 }\end{array}$} & \multicolumn{5}{|c|}{ Dew Point Temperature $\left({ }^{\circ} \mathrm{F}\right)$} & \multicolumn{5}{|c|}{ Wet Bulb Temperature ( $\left.{ }^{\circ} \mathrm{F}\right)$} & \multicolumn{5}{|c|}{ Relative Humidity (\%) } & \multicolumn{7}{|c|}{ Wet Bulb Globe Temperature ( $\left.{ }^{\circ} \mathrm{F}\right)$} \\
\hline & \multirow[b]{2}{*}{ 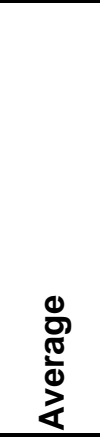 } & \multirow[b]{2}{*}{ 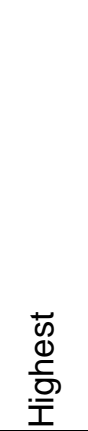 } & \multirow[b]{2}{*}{ 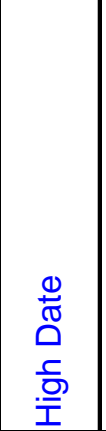 } & \multirow[b]{2}{*}{$\begin{array}{l}\varpi \\
0 \\
0 \\
0\end{array}$} & \multirow[b]{2}{*}{$\begin{array}{l}\stackrel{0}{\tilde{\pi}} \\
0 \\
3 \\
0 \\
0\end{array}$} & \multirow[b]{2}{*}{ 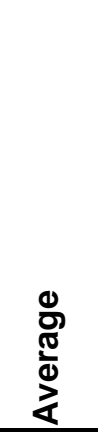 } & \multirow[b]{2}{*}{ 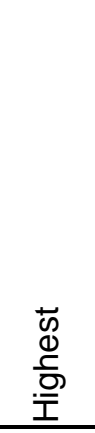 } & \multirow[b]{2}{*}{ 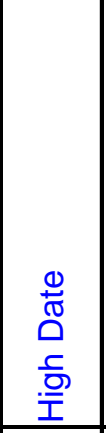 } & \multirow[b]{2}{*}{$\begin{array}{l}\bar{\omega} \\
\bigotimes \\
\vdots \\
\end{array}$} & \multirow[b]{2}{*}{\begin{tabular}{l}
0 \\
\multirow{0}{0}{} \\
0 \\
3 \\
0 \\
\end{tabular}} & \multirow{2}{*}{ 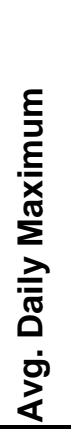 } & \multirow{2}{*}{ 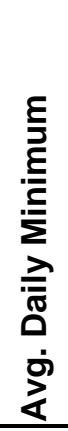 } & \multirow[b]{2}{*}{ 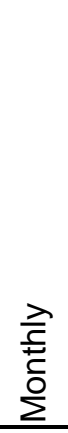 } & \multirow[b]{2}{*}{ 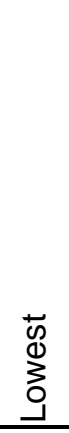 } & \multirow[b]{2}{*}{ 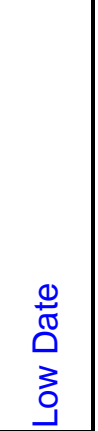 } & \multirow[b]{2}{*}{ 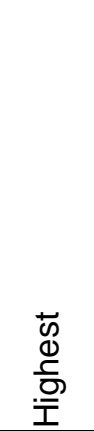 } & \multirow[b]{2}{*}{ 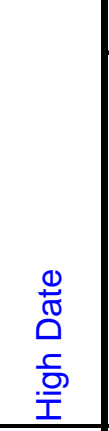 } & \multicolumn{5}{|c|}{ Number of Days } \\
\hline & & & & & & & & & & & & & & & & & & 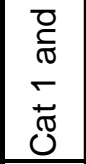 & $\begin{array}{l}\bar{O} \\
\text { c } \\
\sim \\
\sim \\
\tilde{\sigma} \\
\tilde{U}\end{array}$ & 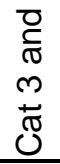 & 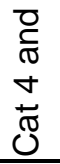 & $\begin{array}{l}0 \\
\mathbb{\pi} \\
0\end{array}$ \\
\hline Jan & 30.3 & 62.5 & 5 th & 10.0 & 8th & 38.2 & 66.9 & 5 th & 18.6 & 8th & 79 & 43 & 62 & 20 & 8th & 73.4 & 5 th & 0 & 0 & 0 & 0 & 0 \\
\hline Feb & 33.3 & 65.0 & 6th & 13.2 & $1 \mathrm{st}$ & 40.2 & 65.1 & 6th & 23.1 & 8th & 82 & 47 & 66 & 19 & $21 s t$ & 69.2 & 6th & 0 & 0 & 0 & 0 & 0 \\
\hline Mar & 40.8 & 61.3 & 5th & 13.9 & 23rd & 49.9 & 67.4 & 5 th & 26.0 & $23 r d$ & 73 & 33 & 55 & 17 & 12th & 78.0 & 28th & 2 & 0 & 0 & 0 & 0 \\
\hline Apr & 44.9 & 63.0 & 11th & 18.2 & 4th & 53.7 & 68.4 & 26th & 29.4 & 5th & 77 & 31 & 54 & 16 & 4th & 80.9 & 24th & 8 & 0 & 0 & 0 & 0 \\
\hline May & 59.7 & 70.8 & 23rd & 34.4 & 4th & 65.1 & 74.6 & 28th & 40.0 & 4th & 83 & 42 & 65 & 29 & 7th & 91.2 & 20th & 28 & 18 & 12 & 1 & 1 \\
\hline Jun & 67.0 & 75.5 & 15th & 48.0 & $1 s t$ & 70.7 & 78.0 & 18th & 60.7 & 2nd & 92 & 47 & 73 & 28 & 7th & 90.7 & 16th & 30 & 29 & 18 & 8 & 3 \\
\hline Jul & 68.1 & 73.6 & $3 r d$ & 54.1 & 16th & 72.1 & 77.8 & 14th & 61.8 & 16th & 90 & 46 & 71 & 32 & 20th & 91.5 & 12th & 31 & 31 & 29 & 17 & 6 \\
\hline Aug & 66.0 & 74.5 & 2nd & 45.9 & 7th & 70.0 & 77.6 & 2nd & 53.6 & 8th & 90 & 47 & 71 & 31 & 8th & 90.3 & 4th & 30 & 25 & 19 & 5 & 1 \\
\hline Sep & 63.7 & 77.4 & 7th & 43.8 & 20th & 67.3 & 78.1 & $1 \mathrm{st}$ & 50.3 & 20th & 88 & 57 & 74 & 34 & 22nd & 89.2 & $1 \mathrm{st}$ & 25 & 7 & 1 & 1 & 0 \\
\hline Oct & 58.7 & 73.0 & 2nd & 39.1 & 15th & 61.8 & 75.6 & 2nd & 41.1 & 16th & 92 & 57 & 79 & 39 & 17th & 85.6 & 2nd & 10 & 5 & 1 & 0 & 0 \\
\hline Nov & 46.3 & 69.9 & 4th & 22.8 & 15th & 51.2 & 72.2 & 2nd & 28.1 & 15th & 89 & 49 & 72 & 28 & 16th & 80.9 & $1 \mathrm{st}$ & 3 & 0 & 0 & 0 & 0 \\
\hline Dec & 35.4 & 65.6 & 9th & 5.1 & 20th & 41.4 & 67.0 & 9th & 18.3 & 20th & 92 & 40 & 71 & 19 & $21 \mathrm{st}$ & 71.5 & 9th & 0 & 0 & 0 & 0 & 0 \\
\hline Year & 51.2 & 77.4 & 7-Sep & 5.1 & 20-Dec & 56.8 & 78.1 & 1-Sep & 18.3 & 20-Dec & 86 & 45 & 68 & 16 & 4-Apr & 91.5 & 12-Jul & 167 & 115 & 80 & 32 & 11 \\
\hline
\end{tabular}


Table 1(c) - Means and Extremes of SRS Meteorological Data for 2004 (cont'd)

\begin{tabular}{|c|c|c|c|c|c|c|c|c|c|c|c|c|c|c|c|c|c|c|c|c|c|}
\hline \multirow[b]{2}{*}{$\begin{array}{l}5 \\
\stackrel{5}{0} \\
\Sigma \\
\Sigma\end{array}$} & \multicolumn{4}{|c|}{ Wind Speed (mph) } & \multicolumn{5}{|c|}{ Barometric Pressure (mb) } & \multicolumn{6}{|c|}{ Solar Radiation (ly) } & \multicolumn{6}{|c|}{ Degree Days } \\
\hline & 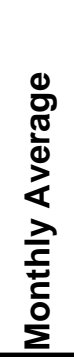 & 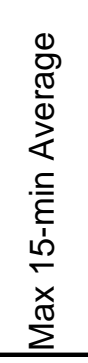 & 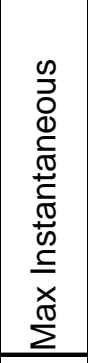 & 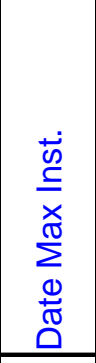 & $\begin{array}{l}\frac{0}{\mathrm{\sigma}} \\
\frac{\pi}{0} \\
\stackrel{0}{\alpha}\end{array}$ & 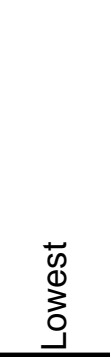 & \begin{tabular}{l}
0 \\
\multirow{\pm}{\pm}{} \\
0 \\
3 \\
0 \\
\end{tabular} & 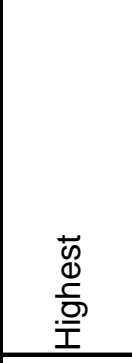 & 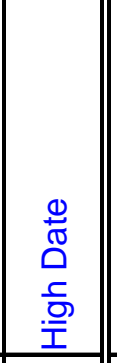 & 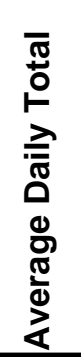 & 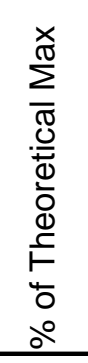 & 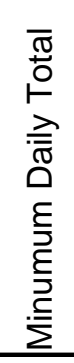 & 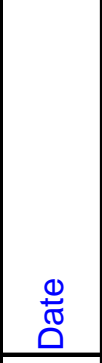 & 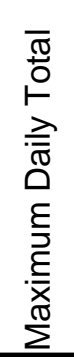 & $\begin{array}{l}\stackrel{0}{ \pm} \\
0 \\
\end{array}$ & 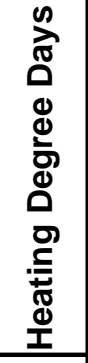 & 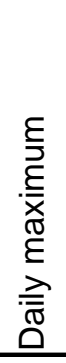 & $\begin{array}{l}\stackrel{0}{ \pm} \\
\text { صै }\end{array}$ & 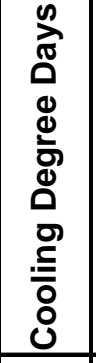 & 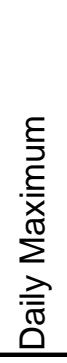 & $\begin{array}{l}\stackrel{0}{ \pm} \\
0 \\
0\end{array}$ \\
\hline Jan & 4.3 & 13.0 & 33.1 & 18th & 1009.0 & 990.5 & 18th & 1023.5 & 7th & 251 & 0.70 & 28 & 25th & 377 & 28th & 663 & 34 & 26th & 2 & 2 & 5th \\
\hline Feb & 4.9 & 18.0 & 34.8 & 7th & 1010.4 & 997.6 & 6th & 1023.8 & 9th & 240 & 0.53 & 32 & 14th & 497 & 28th & 575 & 30 & 27th & 0 & 0 & \\
\hline Mar & 4.8 & 24.4 & 49.9 & 8th & 1011.3 & 996.8 & 16th & 1024.2 & 24th & 429 & 0.75 & 96 & 30th & 575 & 23rd & 212 & 21 & 24th & 11 & 4 & 6th \\
\hline Apr & 4 & 16.9 & 32.0 & 2nd & 1005.4 & 992.6 & 8th & 1019.9 & 18th & 537 & 0.79 & 236 & 30th & 676 & 28th & 110 & 17 & 2nd & 62 & 9 & 26th \\
\hline May & 4.1 & 17.1 & 38.3 & 2nd & 1006.7 & 996.8 & $31 \mathrm{st}$ & 1016.2 & 17th & 544 & 0.72 & 271 & 17th & 691 & 4th & 12 & 8 & 5th & 286 & 17 & 29th \\
\hline Jun & 4.0 & 14.8 & 36.8 & 7th & 1006.5 & 997.2 & $1 s t$ & 1012.2 & 9th & 505 & 0.64 & 167 & $21 \mathrm{st}$ & 668 & 2nd & 0 & 0 & & 381 & 20 & 13th \\
\hline Jul & 3.7 & 13.6 & 34.8 & 4th & 1004.1 & 996.2 & 18th & $\mid 1011.2$ & 1st & 531 & 0.69 & 270 & $1 s t$ & 712 & 8th & 0 & 0 & & 467 & 20 & 15th \\
\hline Aug & 3.8 & 11.6 & 37.2 & 13th & 1005.4 & 995.1 & 5th & 1012.4 & 16th & 453 & 0.64 & 215 & 13th & 672 & 7th & 0 & 0 & & 383 & 18 & 5th \\
\hline Sep & 5.3 & 14.5 & 34.5 & 28th & 1005.4 & 990.5 & 27th & 1014.4 & 21st & 366 & 0.60 & 80 & 27th & 576 & $3 r d$ & 1 & 1 & $21 s t$ & 248 & 13 & 17th \\
\hline Oct & 3.2 & 13.5 & 26.6 & 16th & 1006.6 & 990.3 & 15th & 1019.7 & 7th & 286 & 0.57 & 76 & 28th & 452 & 15th & 44 & 9 & 17th & 80 & 12 & $3 r d$ \\
\hline Nov & 3.5 & 15.6 & 36.8 & 25th & 1010.3 & 991.3 & 25th & 1027.4 & 14th & 252 & 0.63 & 72 & 23rd & 392 & 5th & 288 & 24 & 28th & 23 & 8 & 4th \\
\hline Dec & 4.0 & 17.0 & 36.6 & $21 \mathrm{st}$ & 1010.1 & 990.9 & 10th & 1027.0 & 28th & 242 & 0.73 & 69 & 24th & 324 & 15th & 600 & 34 & $21 \mathrm{st}$ & 4 & 3 & 11th \\
\hline Year & 4.2 & 24.4 & 49.9 & 8-Mar & 1007.6 & 990.3 & 15-Oct & 1027.4 & 14-Nov & 386 & 0.67 & 28 & 25-Jan & 712 & 8-Jul & 2505 & 34 & 21-Dec & 1946 & 20 & 15-Jul \\
\hline
\end{tabular}


Table 2. Monthly and Annual Average and Extreme Temperatures, 1974-2004

\begin{tabular}{|c|c|c|c|c|c|c|c|c|c|c|c|c|c|}
\hline Year & Jan & Feb & Mar & Apr & May & Jun & Jul & Aug & Sep & Oct & Nov & $\mathrm{Dec}$ & Annual \\
\hline 1974 & 59.6 & 50.8 & 62.2 & 66.2 & 75.3 & 77.5 & 81.5 & 80.9 & 75.3 & 64.5 & 56.6 & 49.0 & 66.6 \\
\hline 1975 & 51.4 & 53.2 & 55.8 & 63.9 & 75.6 & 79.1 & 79.7 & 82.4 & 75.7 & 68.7 & 59.3 & 48.5 & 66.1 \\
\hline 1976 & 44.2 & 55.7 & 61.5 & 64.8 & 68.9 & 75.6 & 80.4 & 78.0 & 73.1 & 60.1 & 48.7 & 44.8 & 63.0 \\
\hline 1977 & 35.3 & 47.1 & 60.0 & 66.9 & 73.3 & 80.6 & 83.6 & 80.6 & 77.9 & 62.1 & 58.2 & 46.7 & 64.4 \\
\hline 1978 & 39.3 & 41.3 & 54.2 & 65.7 & 70.9 & 79.7 & 82.1 & 81.2 & 77.1 & 65.6 & 60.7 & 49.6 & 64.0 \\
\hline 1979 & 42.1 & 44.6 & 57.5 & 64.5 & 71.3 & 75.1 & 79.6 & 80.5 & 73.4 & 64.8 & 57.4 & 47.4 & 63.2 \\
\hline 1980 & 45.9 & 44.3 & 52.6 & 63.5 & 71.2 & 78.3 & 83.8 & 82.5 & 79.2 & 62.7 & 52.8 & 46.0 & 63.6 \\
\hline 1981 & 40.4 & 48.5 & 53.0 & 67.0 & 68.6 & 81.3 & 81.3 & 76.3 & 74.0 & 62.1 & 54.4 & 43.2 & 62.5 \\
\hline 1982 & 43.0 & 50.0 & 58.9 & 62.4 & 75.7 & 78.8 & 80.9 & 80.1 & 75.0 & 66.2 & 58.7 & 54.8 & 65.4 \\
\hline 1983 & 43.3 & 48.0 & 55.3 & 59.4 & 66.8 & 76.7 & 84.3 & 83.9 & 74.8 & 67.2 & 56.4 & 45.8 & 63.5 \\
\hline 1984 & 45.0 & 51.7 & 56.5 & 62.6 & 71.9 & 80.1 & 80.1 & 80.8 & 74.0 & 73.4 & 53.4 & 56.9 & 65.5 \\
\hline 1985 & 42.9 & 49.5 & 60.2 & 67.5 & 74.5 & 80.8 & 81.1 & 79.7 & 75.7 & 70.8 & 65.5 & 45.4 & 66.1 \\
\hline 1986 & 45.4 & 54.6 & 57.9 & 66.4 & 74.4 & 82.7 & 86.9 & 80.1 & 78.4 & 67.1 & 61.3 & 49.3 & 67.0 \\
\hline 1987 & 46.2 & 48.6 & 56.5 & 62.3 & 74.5 & 79.9 & 82.8 & 83.8 & 76.6 & 60.7 & 59.1 & 52.9 & 65.3 \\
\hline 1988 & 42.3 & 47.8 & 56.8 & 64.2 & 70.4 & 76.8 & 81.6 & 81.4 & 75.4 & 61.2 & 58.0 & 49.1 & 63.8 \\
\hline 1989 & 52.2 & 52.0 & 58.3 & 64.2 & 70.6 & 79.8 & 81.4 & 80.9 & 75.3 & 67.3 & 52.4 & 44.2 & 64.9 \\
\hline 1990 & 54.9 & 57.5 & 60.0 & 64.0 & 72.9 & 80.5 & 83.7 & 83.8 & 79.0 & 69.4 & 59.9 & 54.6 & 68.4 \\
\hline 1991 & 47.9 & 54.1 & 60.3 & 69.2 & 76.9 & 79.5 & 83.6 & 81.2 & 77.4 & 68.1 & 55.4 & 54.0 & 67.3 \\
\hline 1992 & 49.5 & 54.1 & 57.2 & 65.0 & 71.2 & 78.9 & 83.7 & 80.7 & 76.9 & 65.0 & 57.1 & 48.0 & 65.6 \\
\hline 1993 & 51.7 & 47.8 & 53.2 & 58.9 & 69.7 & 78.2 & 83.6 & 80.0 & 75.2 & 62.8 & 55.2 & 43.6 & 63.3 \\
\hline 1994 & 41.5 & 50.1 & 60.2 & 68.0 & 71.2 & 82.3 & 81.8 & 81.2 & 77.4 & 67.2 & 62.3 & 53.3 & 66.4 \\
\hline 1995 & 45.5 & 49.9 & 58.6 & 65.9 & 73.5 & 75.0 & 79.9 & 79.0 & 71.8 & 65.9 & 50.8 & 43.8 & 63.3 \\
\hline 1996 & 44.6 & 50.1 & 50.6 & 61.6 & 72.9 & 76.5 & 79.3 & 76.0 & 72.7 & 62.1 & 51.6 & 48.8 & 62.2 \\
\hline 1997 & 48.2 & 52.9 & 63.3 & 61.2 & 68.5 & 74.0 & 80.2 & 79.0 & 75.0 & 64.1 & 51.6 & 47.0 & 63.8 \\
\hline 1998 & 49.7 & 51.1 & 53.6 & 62.7 & 74.6 & 82.1 & 82.6 & 80.3 & 75.8 & 66.9 & 60.5 & 53.6 & 66.1 \\
\hline 1999 & 51.9 & 51.6 & 53.4 & 67.2 & 69.7 & 76.6 & 80.7 & 82.9 & 73.8 & 64.3 & 58.1 & 48.6 & 64.9 \\
\hline 2000 & 44.4 & 50.2 & 58.5 & 60.7 & 75.1 & 78.0 & 79.9 & 77.6 & 71.7 & 62.5 & 53.1 & 38.2 & 62.5 \\
\hline 2001 & 43.8 & 52.4 & 53.0 & 63.9 & 71.3 & 75.3 & 77.7 & 78.8 & 71.2 & 62.2 & 60.0 & 52.4 & 63.5 \\
\hline 2002 & 47.3 & 48.0 & 57.6 & 68.1 & 70.2 & 77.5 & 80.5 & 78.4 & 75.4 & 66.7 & 51.7 & 44.5 & 63.8 \\
\hline 2003 & 42.0 & 47.5 & 57.6 & 61.6 & 70.6 & 75.2 & 77.3 & 77.7 & 71.9 & 63.7 & 58.2 & 42.9 & 62.2 \\
\hline 2004 & 43.7 & 45.2 & 58.5 & 63.4 & 74.0 & 77.7 & 80.1 & 77.3 & 73.2 & 66.2 & 56.1 & 45.8 & 63.4 \\
\hline
\end{tabular}

\begin{tabular}{|c|c|c|c|c|c|c|c|c|c|c|c|c|c|}
\hline Avg & 46.0 & 50.0 & 57.2 & 64.3 & 72.1 & 78.4 & 81.5 & 80.2 & 75.1 & 65.2 & 56.6 & 48.2 & 64.6 \\
\hline Lowest Mon & 35.3 & 41.3 & 50.6 & 58.9 & 66.8 & 74.0 & 77.3 & 76.0 & 71.2 & 60.1 & 48.7 & 38.2 & 62.2 \\
\hline Yr Lowest & 1977 & 1978 & 1971 & 1993 & 1983 & 1997 & 1967 & 1973 & 1973 & 1976 & 1976 & 2000 & 1996 \\
\hline Rec Low & -3 & 10 & 11 & 29 & 38 & 48 & 56 & 56 & 41 & 28 & 18 & 5 & -3 \\
\hline Yr Rec & 1985 & 1996 & 1980 & 1983 & 1989 & 1984 & 1963 & 1986 & 1967 & 1976 & 1970 & 1962 & 1985 \\
\hline Highest Mon & 59.6 & 57.5 & 63.3 & 69.2 & 76.9 & 82.7 & 86.9 & 83.9 & 79.2 & 73.4 & 65.5 & 56.9 & 68.4 \\
\hline Yr Highest & 1974 & 1990 & 1997 & 1991 & 1991 & 1986 & 1986 & 1983 & 1980 & 1984 & 1985 & 1971 & 1990 \\
\hline Rec High & 86 & 86 & 90 & 99 & 102 & 105 & 107 & 107 & 104 & 96 & 89 & 82 & 107 \\
\hline Yr Rec & 1975 & 1989 & 1974 & 1986 & 1963 & 1985 & 1986 & 1983 & 1990 & 1986 & 1987 & 1984 & 1986 \\
\hline
\end{tabular}


Table 3. Monthly and Annual Rainfall, 1974-2004

\begin{tabular}{|c|c|c|c|c|c|c|c|c|c|c|c|c|c|}
\hline Year & Jan & Feb & Mar & Apr & May & Jun & Jul & Aug & Sep & Oct & Nov & Dec & Annual \\
\hline 1974 & 2.42 & 6.66 & 3.03 & 3.05 & 3.35 & 2.80 & 4.44 & 6.77 & 3.32 & 0.09 & 1.99 & 4.11 & 42.03 \\
\hline 1975 & 4.98 & 6.64 & 5.92 & 4.42 & 5.15 & 3.83 & 8.55 & 3.83 & 5.18 & 1.74 & 3.41 & 2.03 & 55.68 \\
\hline 1976 & 4.18 & 1.08 & 3.83 & 2.50 & 10.90 & 4.35 & 1.95 & 1.64 & 5.48 & 4.92 & 4.19 & 5.08 & 50.10 \\
\hline 1977 & 3.72 & 1.62 & 6.86 & 1.27 & 1.79 & 2.47 & 3.42 & 7.30 & 5.50 & 4.27 & 1.63 & 3.86 & 43.71 \\
\hline 1978 & 10.02 & 1.31 & 3.06 & 3.53 & 3.64 & 3.42 & 4.11 & 5.10 & 4.06 & 0.06 & 3.54 & 1.87 & 43.72 \\
\hline 1979 & 3.59 & 7.74 & 3.09 & 6.49 & 8.94 & 1.54 & 7.85 & 2.12 & 6.13 & 1.35 & 3.95 & 2.17 & 54.96 \\
\hline 1980 & 5.12 & 3.48 & 10.96 & 1.69 & 3.49 & 2.99 & 0.90 & 2.03 & 5.86 & 2.14 & 2.50 & 1.91 & 43.07 \\
\hline 1981 & 0.89 & 5.02 & 4.72 & 2.07 & 6.90 & 4.29 & 3.96 & 5.79 & 0.54 & 2.81 & 1.00 & 9.55 & 47.54 \\
\hline 1982 & 3.94 & 4.46 & 2.51 & 5.68 & 2.73 & 4.28 & 11.49 & 5.02 & 4.62 & 3.87 & 2.41 & 4.85 & 55.86 \\
\hline 1983 & 3.75 & 7.22 & 6.62 & 5.77 & 1.67 & 6.57 & 4.85 & 6.32 & 3.56 & 1.92 & 5.39 & 4.15 & 57.79 \\
\hline 1984 & 3.51 & 7.09 & 6.05 & 8.00 & 9.79 & 2.54 & 7.28 & 5.52 & 0.60 & 0.31 & 0.90 & 1.38 & 52.97 \\
\hline 1985 & 3.01 & 6.92 & 1.31 & 0.84 & 1.70 & 4.62 & 8.10 & 4.38 & 0.49 & 6.34 & 6.36 & 2.48 & 46.55 \\
\hline 1986 & 1.46 & 3.58 & 4.08 & 1.45 & 3.84 & 3.03 & 2.96 & 10.90 & 1.54 & 4.19 & 5.82 & 5.83 & 48.68 \\
\hline 1987 & 7.39 & 7.55 & 4.97 & 0.70 & 3.57 & 5.64 & 4.87 & 4.93 & 3.56 & 0.29 & 2.74 & 1.42 & 47.63 \\
\hline 1988 & 4.15 & 3.19 & 2.91 & 4.78 & 2.85 & 7.12 & 1.78 & 6.80 & 4.40 & 3.39 & 2.17 & 2.91 & 46.45 \\
\hline 1989 & 1.42 & 3.59 & 5.52 & 4.89 & 2.60 & 6.67 & 11.46 & 3.27 & 4.87 & 3.36 & 3.00 & 4.41 & 55.06 \\
\hline 1990 & 3.07 & 2.38 & 2.37 & 1.21 & 2.95 & 0.89 & 7.31 & 8.07 & 0.62 & 19.62 & 1.41 & 1.57 & 51.47 \\
\hline 1991 & 7.03 & 1.84 & 7.89 & 4.73 & 3.06 & 2.17 & 7.89 & 9.26 & 4.40 & 0.99 & 1.55 & 3.32 & 54.13 \\
\hline 1992 & 4.45 & 3.89 & 2.98 & 2.40 & 1.34 & 6.27 & 3.69 & 4.83 & 6.38 & 3.11 & 7.78 & 2.86 & 49.98 \\
\hline 1993 & 7.45 & 3.62 & 8.37 & 1.74 & 1.43 & 3.27 & 3.12 & 2.23 & 7.29 & 0.99 & 1.87 & 1.81 & 43.19 \\
\hline 1994 & 4.80 & 3.91 & 6.42 & 1.05 & 1.45 & 5.08 & 7.47 & 3.47 & 0.99 & 10.01 & 3.05 & 4.62 & 52.32 \\
\hline 1995 & 6.96 & 7.97 & 0.92 & 1.28 & 1.77 & 8.15 & 5.71 & 6.92 & 5.75 & 2.64 & 2.38 & 4.47 & 54.92 \\
\hline 1996 & 3.65 & 2.43 & 6.64 & 2.40 & 2.96 & 3.04 & 5.57 & 6.91 & 3.67 & 2.16 & 2.32 & 3.20 & 44.95 \\
\hline 1997 & 4.20 & 5.45 & 2.69 & 4.38 & 2.38 & 6.90 & 7.09 & 2.01 & 4.89 & 4.08 & 5.51 & 9.09 & 58.67 \\
\hline 1998 & 7.73 & 8.90 & 6.69 & 7.35 & 4.05 & 4.65 & 5.27 & 2.88 & 4.81 & 0.78 & 0.82 & 1.80 & 55.73 \\
\hline 1999 & 5.31 & 2.29 & 3.44 & 1.95 & 1.26 & 7.52 & 4.91 & 3.14 & 4.46 & 2.57 & 1.50 & 1.21 & 39.56 \\
\hline 2000 & 5.77 & 0.73 & 3.95 & 1.34 & 1.36 & 4.74 & 2.47 & 4.49 & 7.70 & 0.02 & 3.50 & 1.53 & 37.60 \\
\hline 2001 & 3.11 & 2.68 & 7.21 & 1.28 & 3.85 & 6.49 & 4.79 & 3.55 & 3.33 & 0.50 & 1.03 & 0.54 & 38.36 \\
\hline 2002 & 2.85 & 2.13 & 3.86 & 2.58 & 1.69 & 2.30 & 5.95 & 5.47 & 3.45 & 3.19 & 4.00 & 3.58 & 41.05 \\
\hline 2003 & 1.73 & 5.00 & 7.09 & 8.43 & 5.57 & 10.99 & 8.91 & 4.59 & 2.70 & 3.03 & 1.21 & 1.93 & 61.18 \\
\hline 2004 & 2.85 & 6.71 & 0.81 & 1.34 & 3.45 & 6.41 & 1.23 & 2.96 & 10.26 & 1.02 & 3.17 & 2.69 & 42.90 \\
\hline
\end{tabular}

\begin{tabular}{|c|c|c|c|c|c|c|c|c|c|c|c|c|c|}
\hline Avg & 4.34 & 4.42 & 4.73 & 3.24 & 3.60 & 4.68 & 5.46 & 4.92 & 4.21 & 3.09 & 2.97 & 3.30 & 48.96 \\
\hline Min & 0.89 & 0.73 & 0.81 & 0.70 & 1.31 & 0.89 & 0.90 & 1.04 & 0.49 & 0.02 & 0.21 & 0.46 & 28.82 \\
\hline Yr Min & 1981 & 2000 & 2004 & 1972 & 1965 & 1990 & 1980 & 1963 & 1985 & 2000 & 1958 & 1955 & 1954 \\
\hline Max & 10.02 & 8.90 & 10.96 & 8.43 & 10.90 & 10.99 & 11.49 & 10.90 & 10.26 & 19.62 & 7.78 & 9.55 & 73.47 \\
\hline Yr Max & 1978 & 1998 & 1980 & 2003 & 1976 & 2003 & 1982 & 1964 & 2004 & 1990 & 1992 & 1997 & 1964 \\
\hline
\end{tabular}


Table 4 - SRS Rainfall (in inches) for 2004, Manual Gauges

\begin{tabular}{|c|c|c|c|c|c|c|c|c|c|c|c|c|}
\hline Month & 交 & 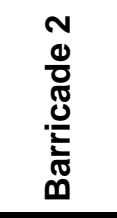 & 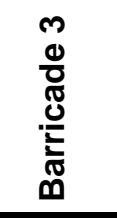 & 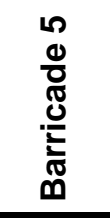 & $\begin{array}{l}\text { Ù } \\
\text { ઠे }\end{array}$ & $\begin{array}{l}\text { Q } \\
\text { ò }\end{array}$ & $\begin{array}{l}4 \\
\text { 오 }\end{array}$ & $\begin{array}{l}\text { I } \\
\text { ᄋ̀ } \\
\text { N }\end{array}$ & $\begin{array}{l}\text { Yे } \\
\text { ᄋ }\end{array}$ & ل’ & 옹 & $\begin{array}{l}0 \\
\frac{v}{\alpha} \\
\dot{r}\end{array}$ \\
\hline Jan & 3.45 & 3.71 & 4.58 & 3.07 & 2.95 & 2.70 & 4.63 & 4.77 & 2.81 & 2.25 & 2.43 & 3.79 \\
\hline Feb & 6.74 & 5.89 & 7.26 & 7.32 & 6.77 & 7.11 & 6.81 & 6.94 & 6.72 & 6.82 & 6.81 & 6.28 \\
\hline Mar & 1.35 & 1.09 & 1.15 & 0.72 & 0.70 & 0.86 & 0.99 & 0.84 & 0.86 & 0.59 & 0.61 & 1.44 \\
\hline Apr & 1.83 & 2.11 & 3.56 & 2.04 & 1.50 & 1.28 & 1.69 & 1.83 & 1.04 & 1.56 & 1.64 & 1.94 \\
\hline May & 2.95 & 4.31 & 2.34 & 3.48 & 4.13 & 2.79 & 2.47 & 2.58 & 5.08 & 3.24 & 2.28 & 2.50 \\
\hline Jun & 8.38 & 11.27 & 13.21 & 8.27 & 6.79 & 7.83 & 8.49 & 10.23 & 9.82 & 4.58 & 4.42 & 8.71 \\
\hline Jul & 4.54 & 4.24 & 1.91 & 3.69 & 3.20 & 2.91 & 3.01 & 2.76 & 4.49 & 3.15 & 2.82 & 4.66 \\
\hline Aug & 2.36 & 5.00 & 4.93 & 6.49 & 3.10 & 2.12 & 4.21 & 6.02 & 3.17 & 3.58 & 5.68 & 2.74 \\
\hline Sep & 8.66 & 8.08 & 7.67 & 7.19 & 9.36 & 7.05 & 10.54 & 10.68 & 9.53 & 9.88 & 8.90 & 8.72 \\
\hline Oct & 0.37 & 0.82 & 1.05 & 1.21 & 1.19 & 0.59 & 3.32 & 1.37 & 1.25 & 1.19 & 0.93 & 0.66 \\
\hline Nov & 4.61 & 4.76 & 5.29 & 4.74 & 4.01 & 2.74 & 4.11 & 4.25 & 2.91 & 3.09 & 3.00 & 4.74 \\
\hline Dec & 1.67 & 2.43 & 3.67 & 2.16 & 2.10 & 2.54 & 3.81 & 2.80 & 1.94 & 1.74 & 1.89 & 1.72 \\
\hline Annual & 46.91 & 53.71 & 56.62 & 50.38 & 45.80 & 40.52 & 54.08 & 55.07 & 49.62 & 41.67 & 41.41 & 47.90 \\
\hline
\end{tabular}


Fig. 1 SRS Meteorological Monitoring Network

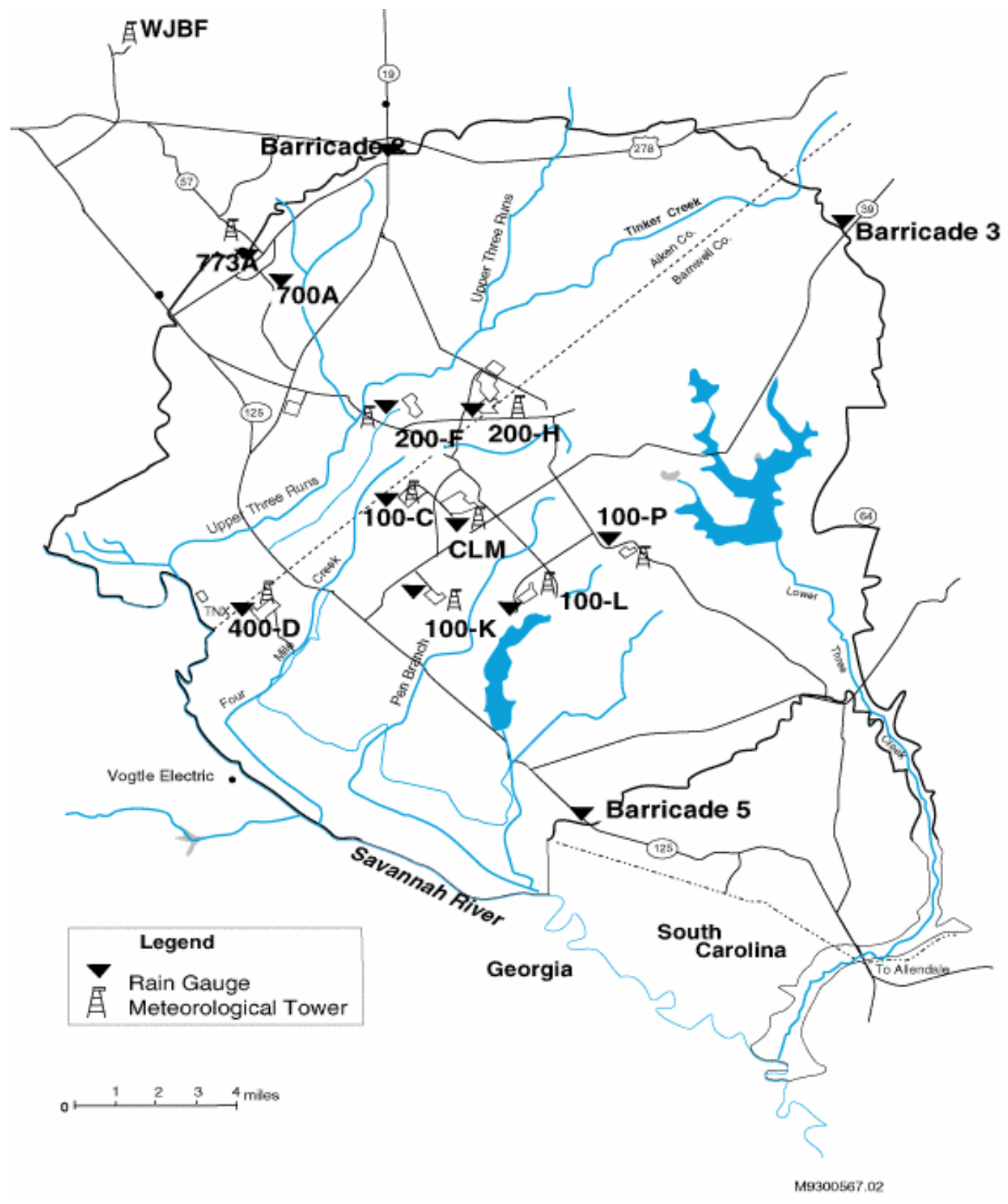


Fig. 2 - Summary of Daily Data for 2004
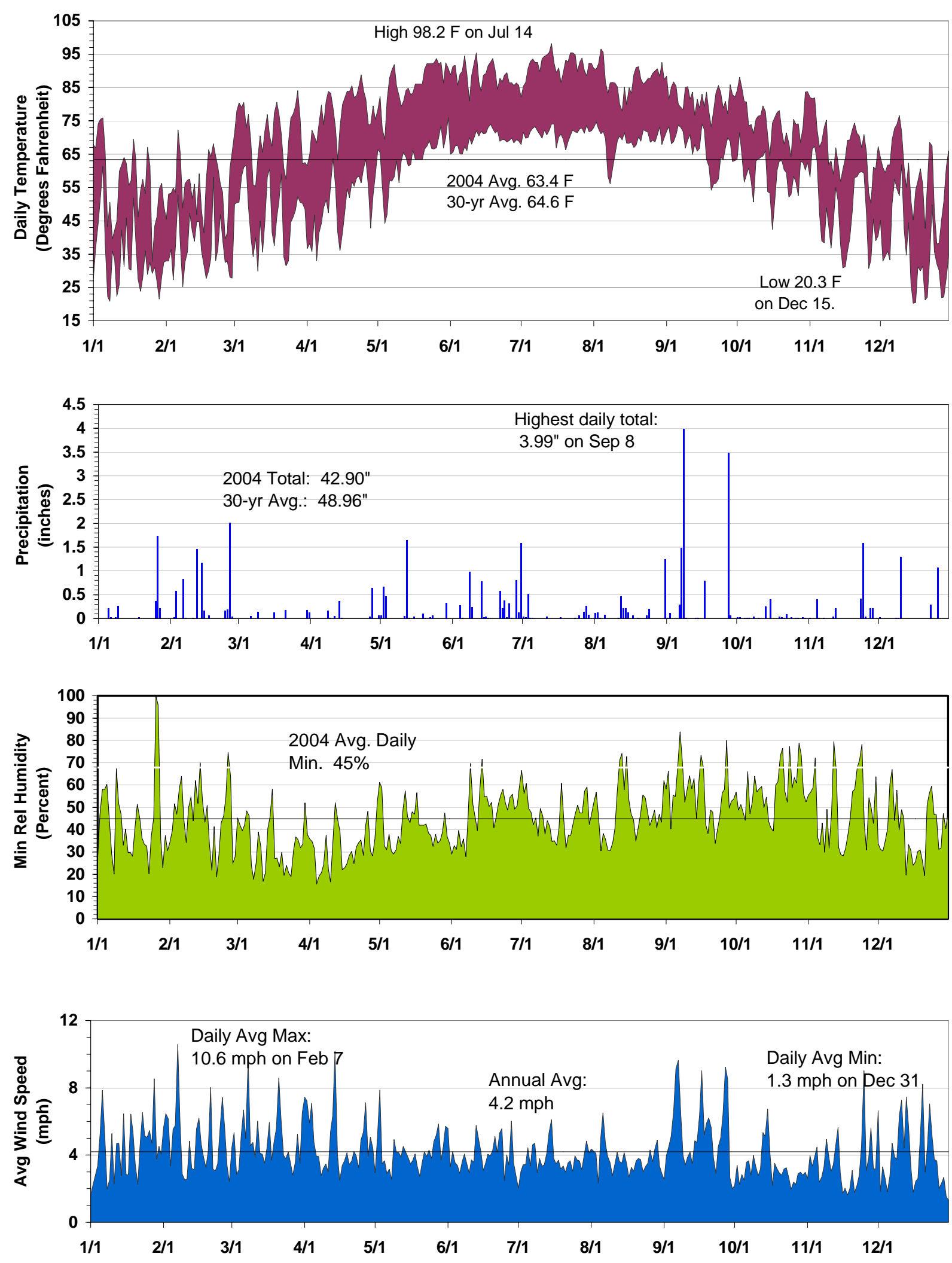
Fig. 3 - Daily High and Low Temperatures for 2004

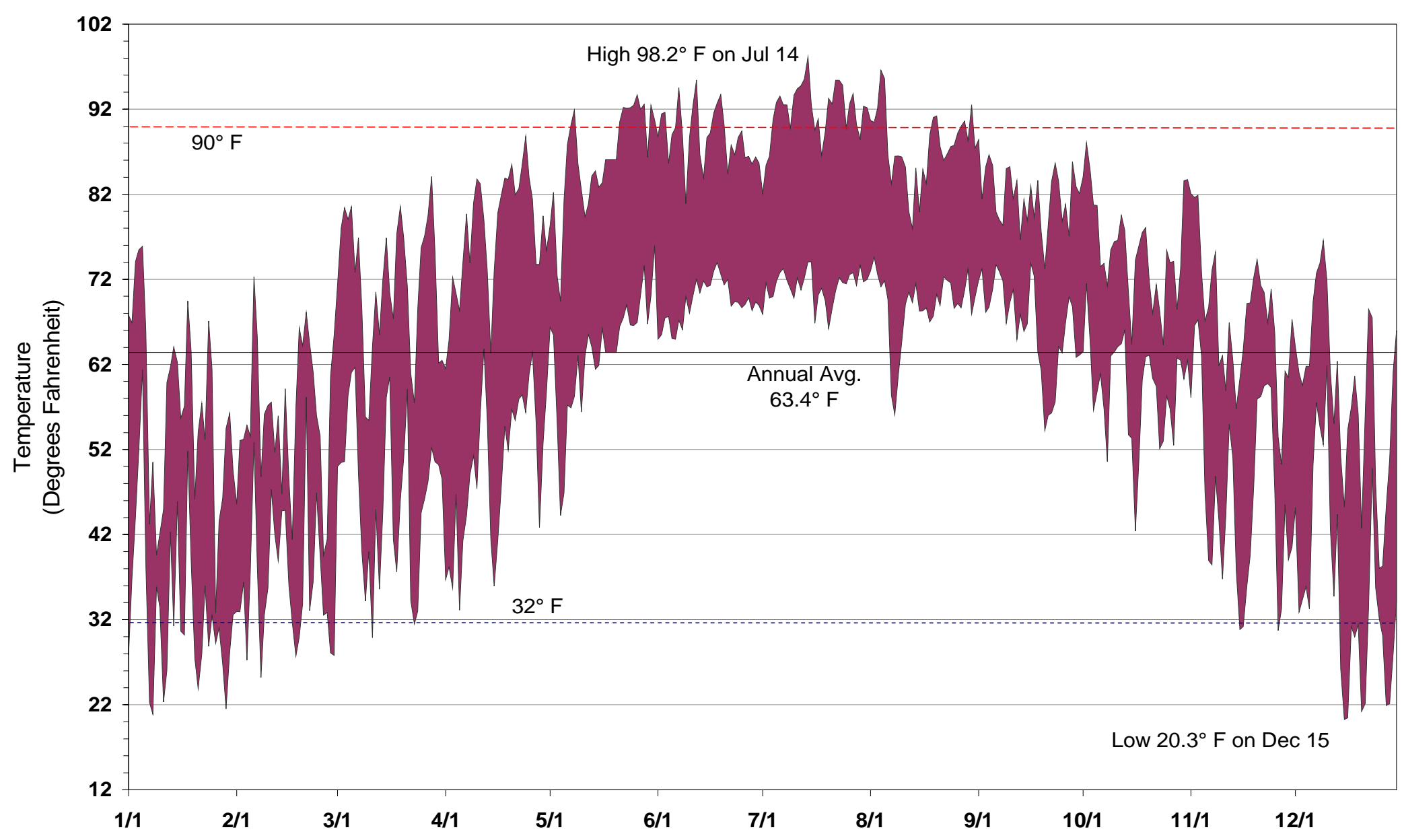


Fig. 4 - SRS Annual Average Temperature 1974-2004

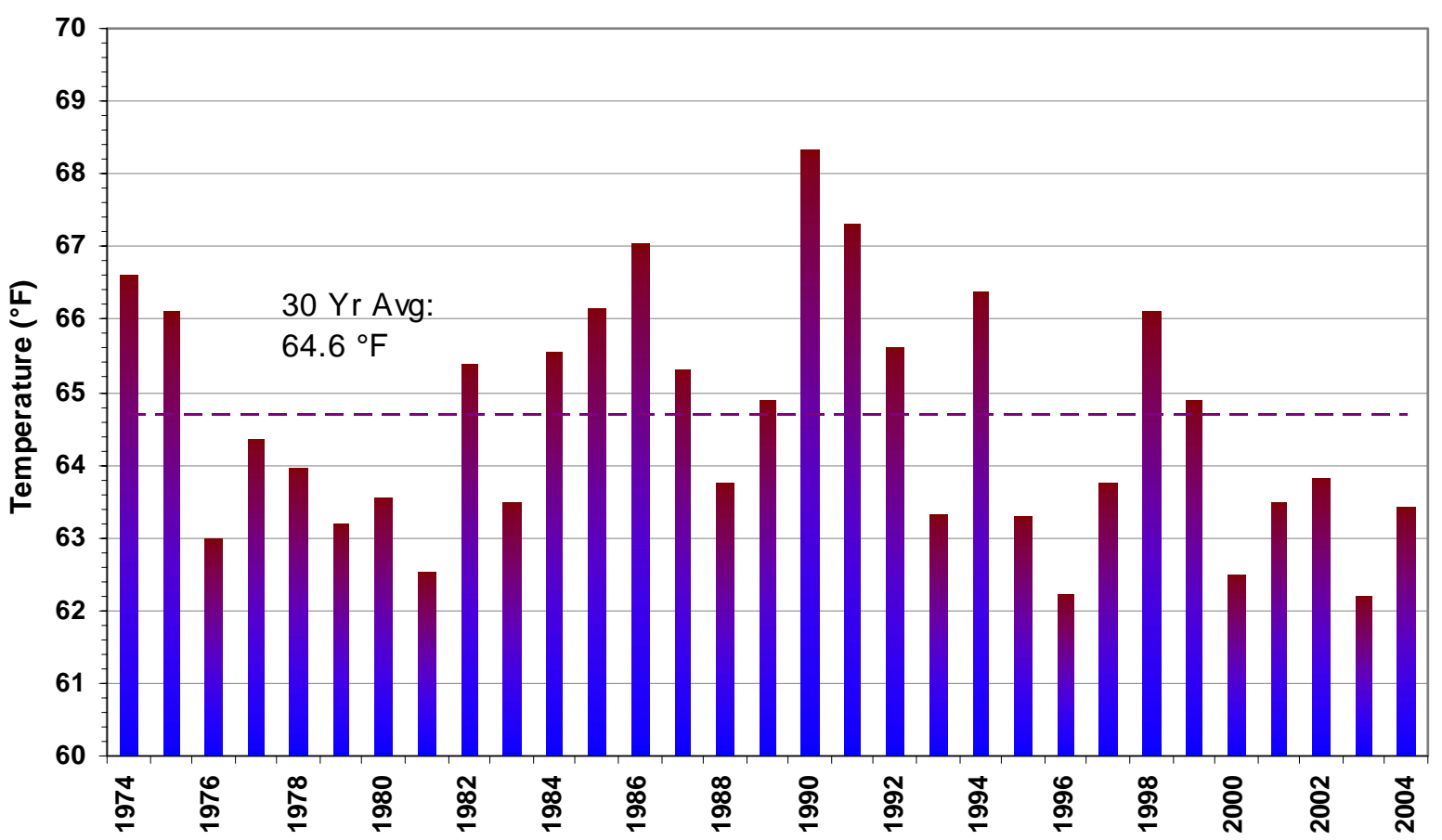

Fig. 5 - SRS Monthly Average Tem perature

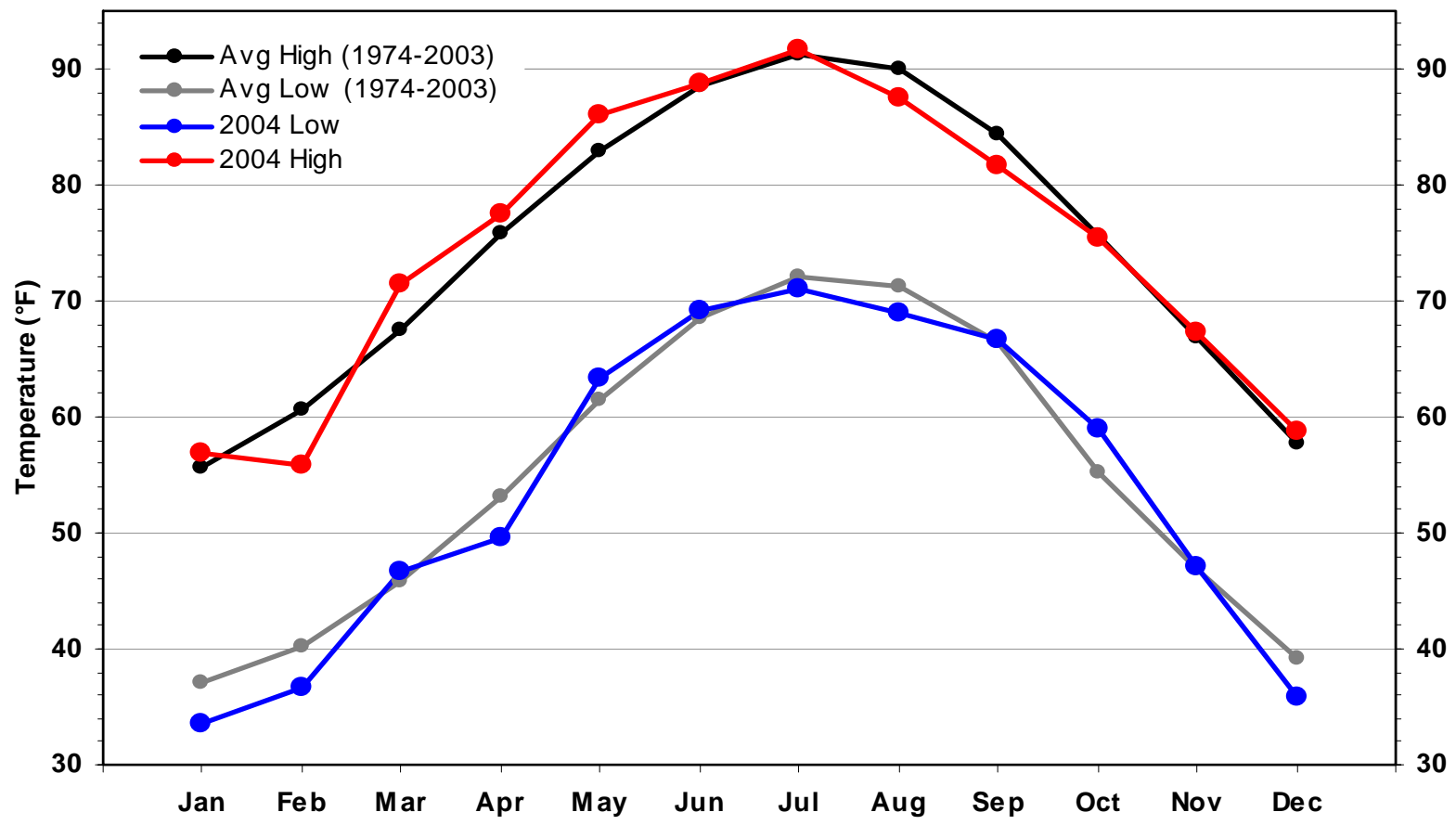




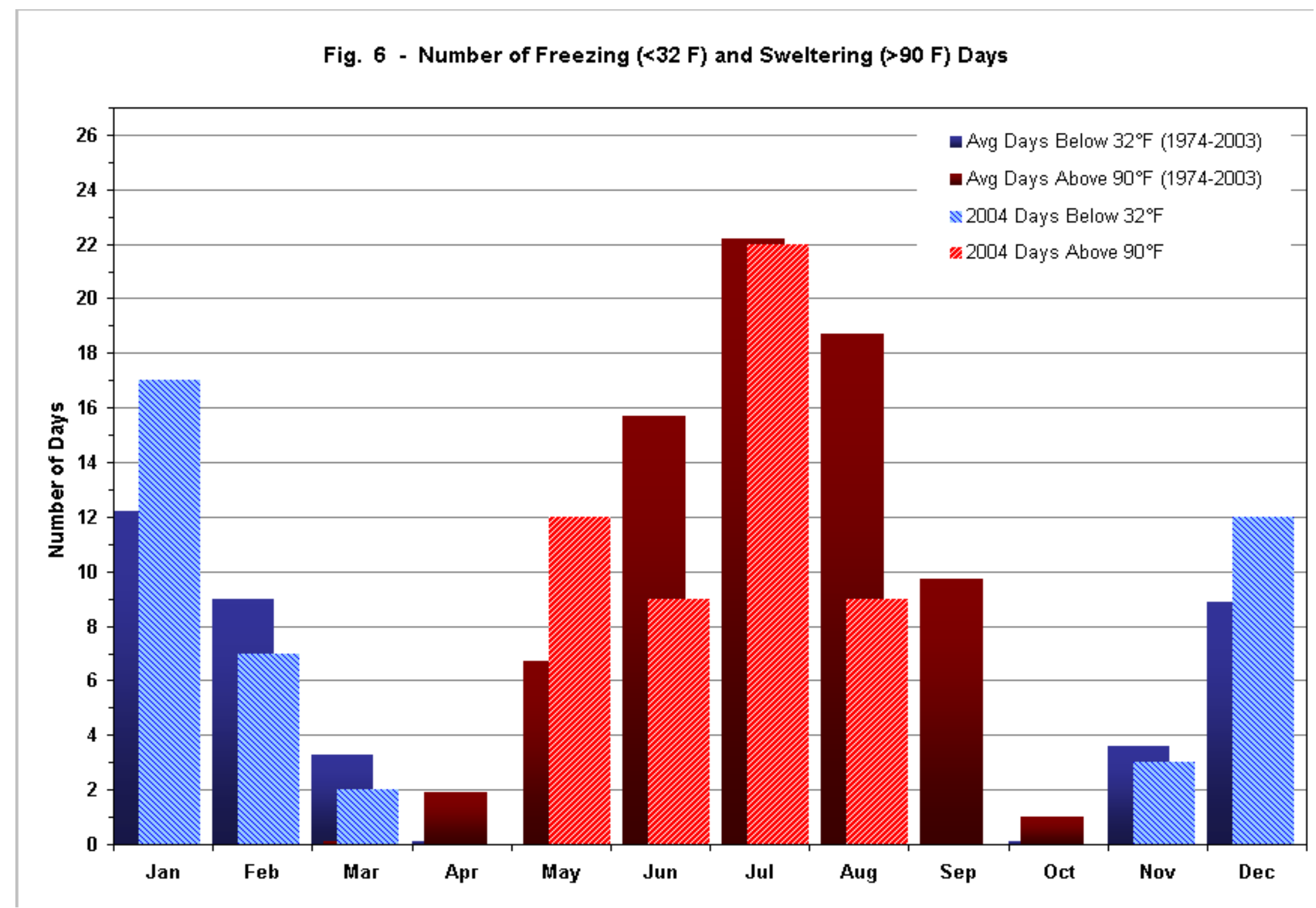




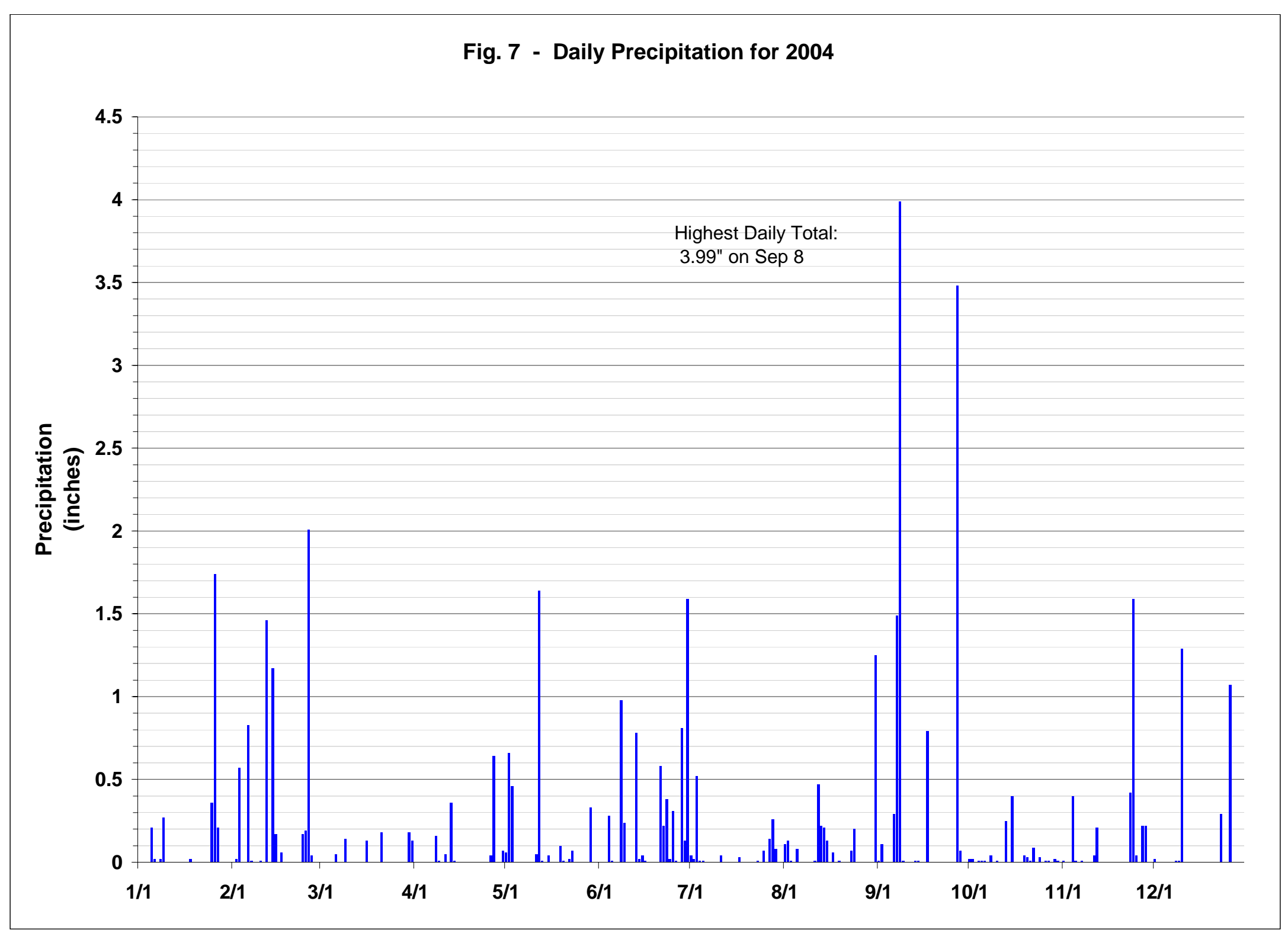


Fig. 8 - SRS Annual Precipitation 1974-2004

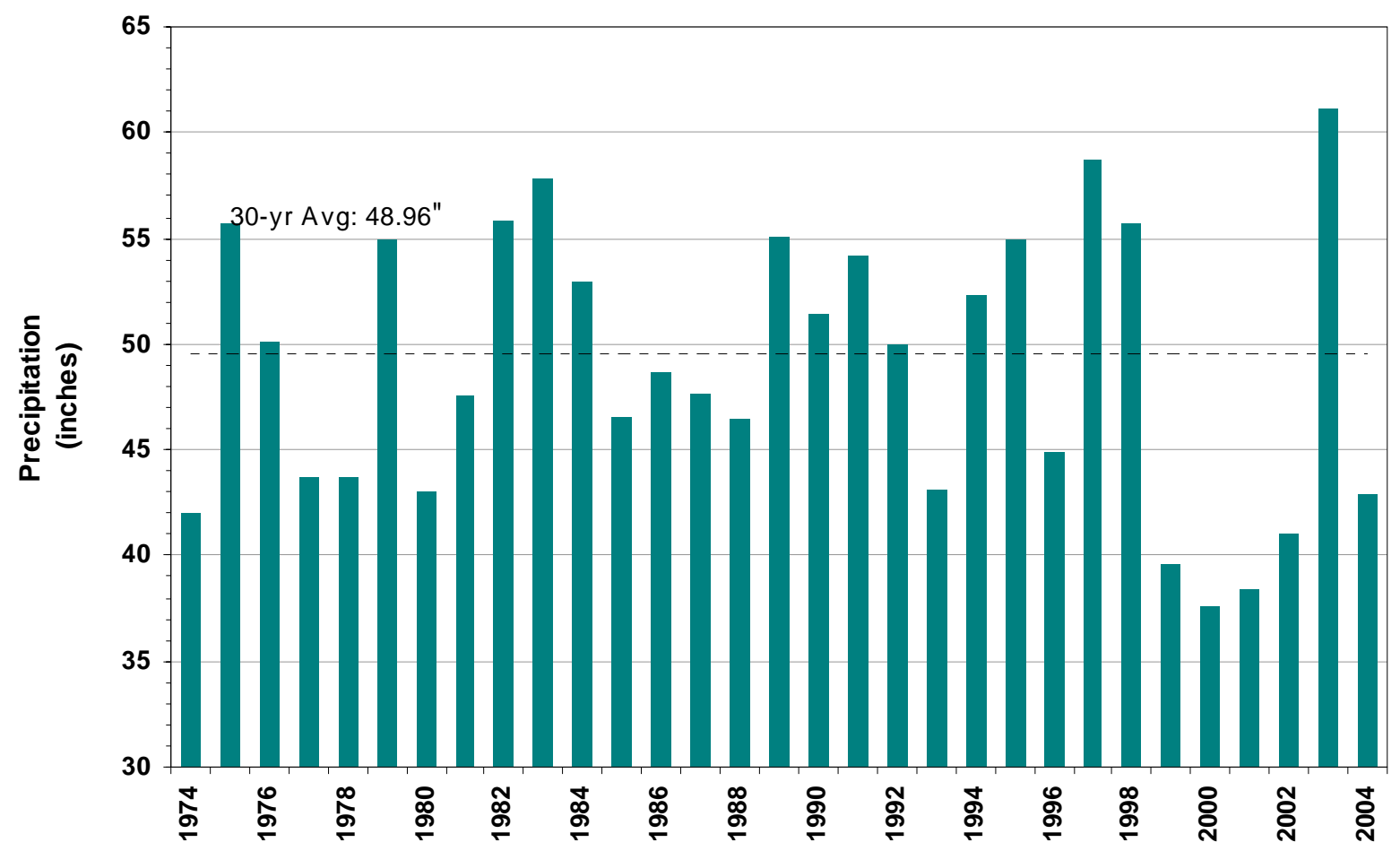

Fig. 9 - SRS Monthly Precipitation

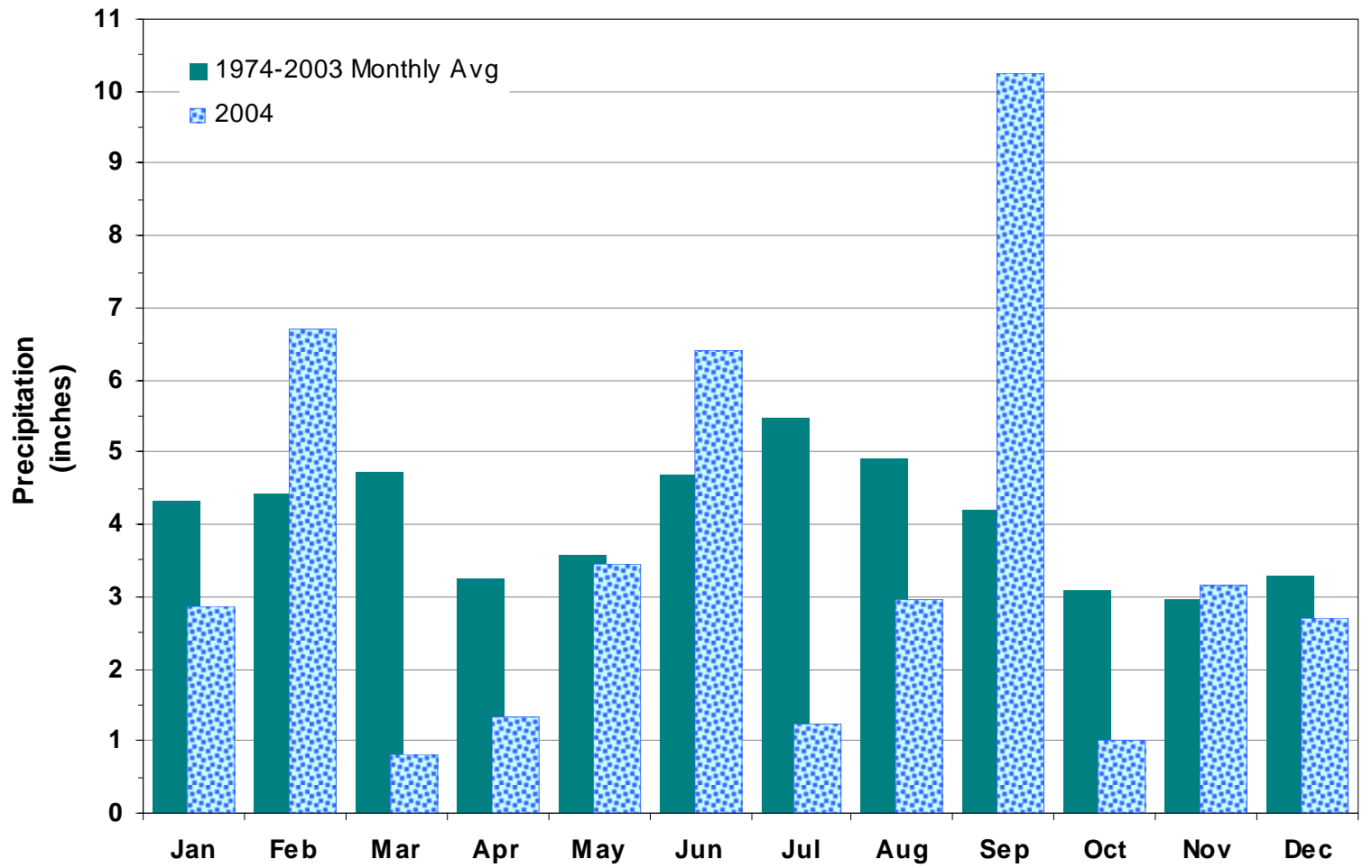


Fig. 10 - Daily High and Low Humidity for 2004

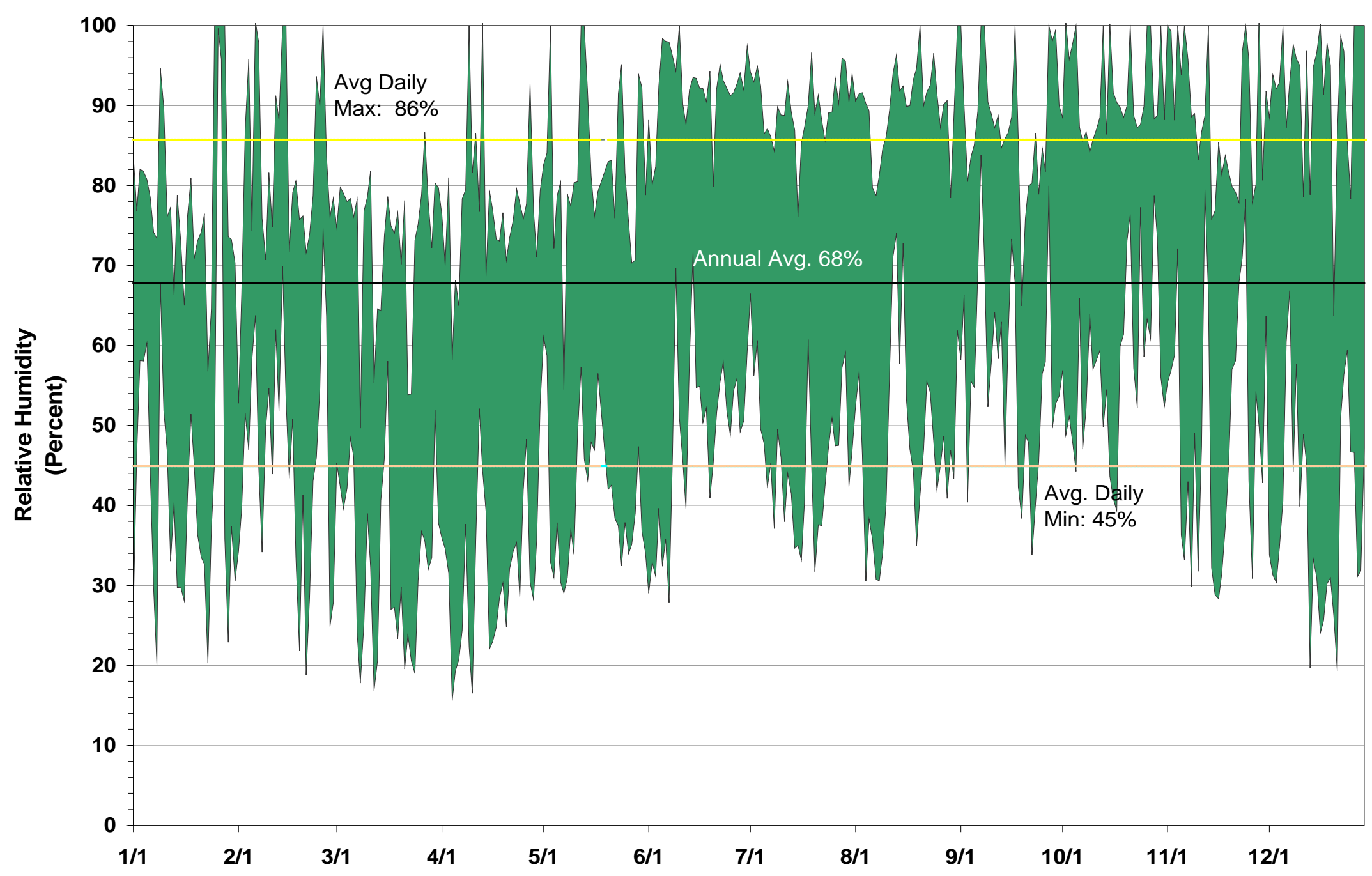


Fig. 11 - SRS Annual Average Humidity 1974-2004

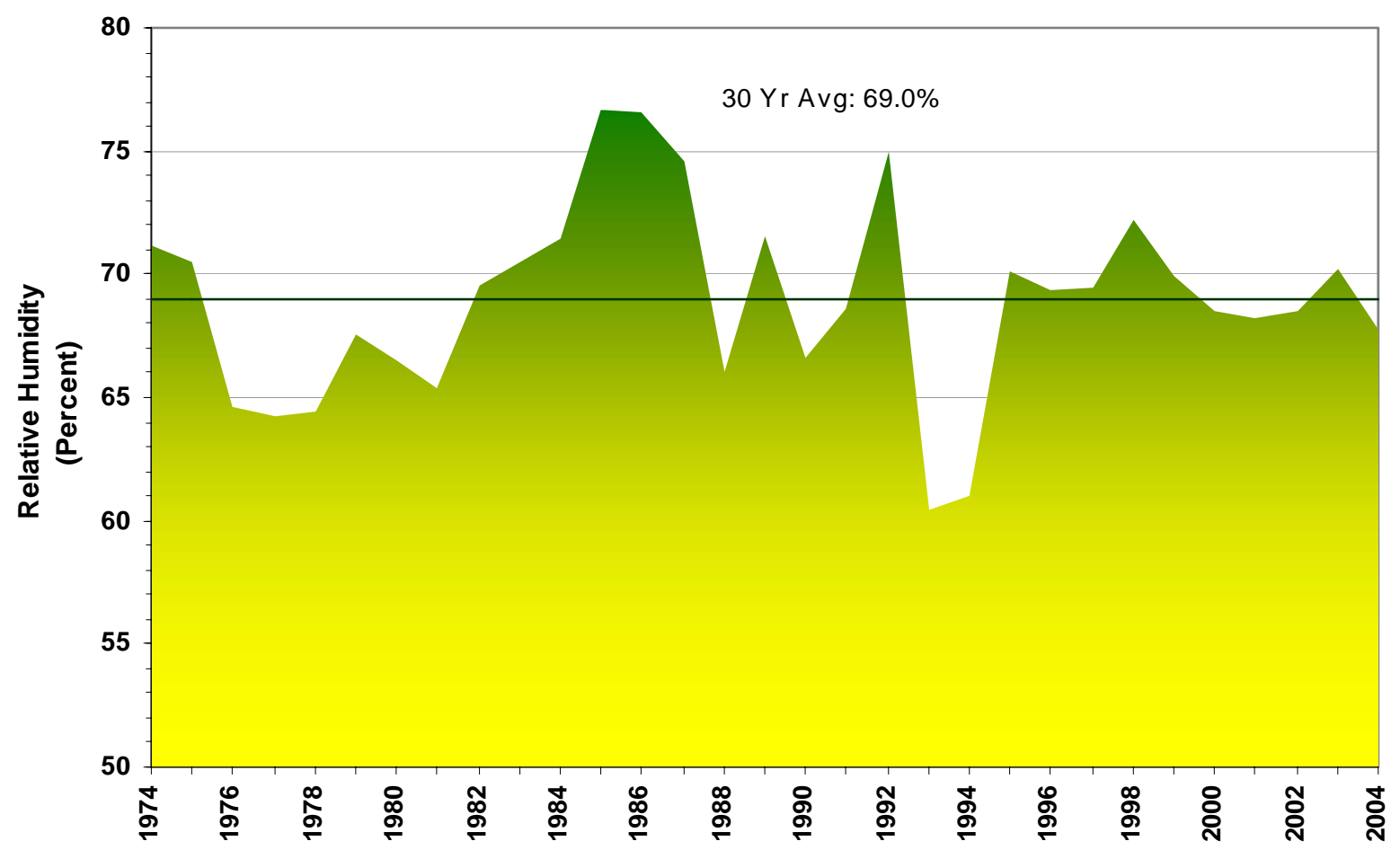

Fig. 12 - SRS Monthly Average Minimum Humidity

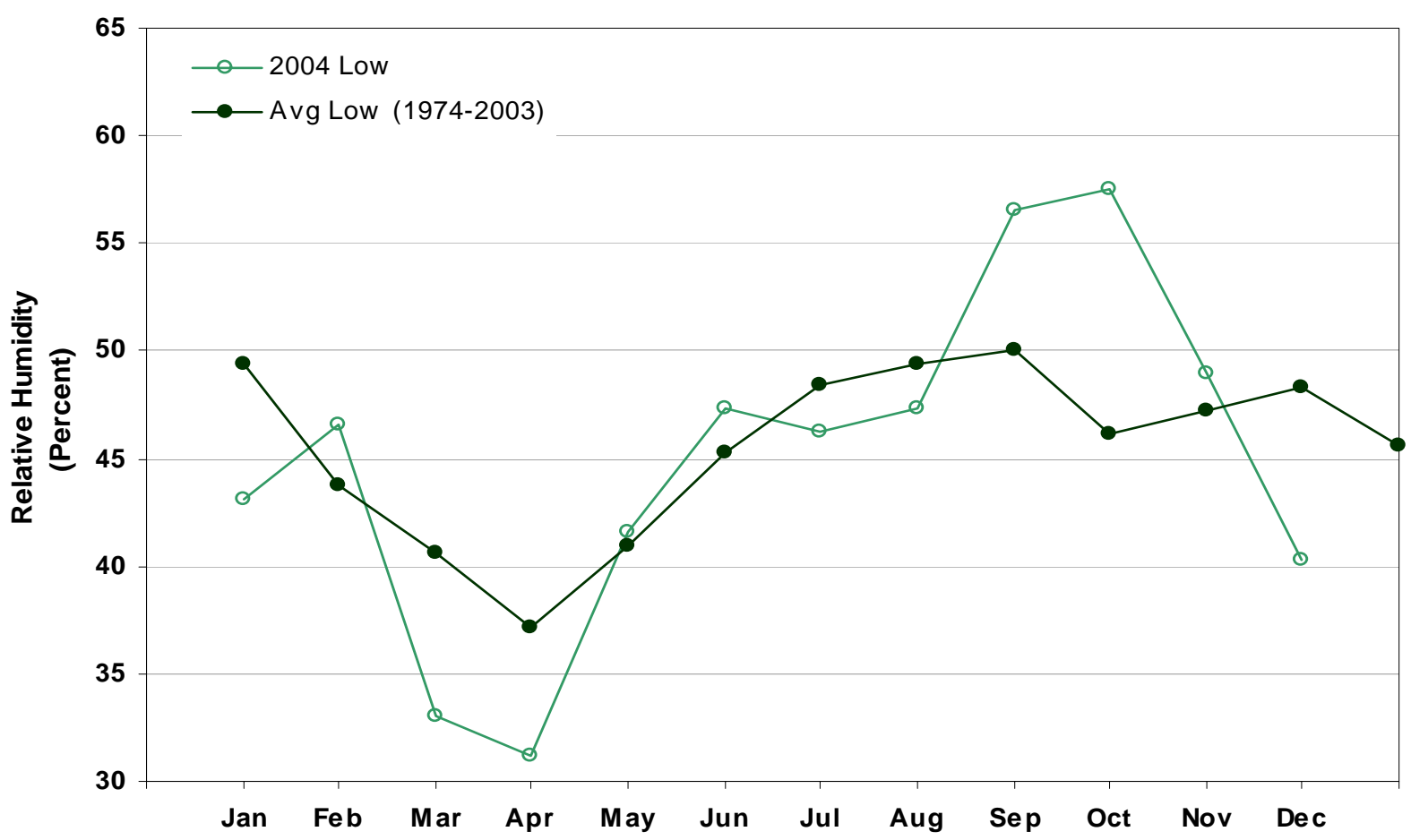


Fig. 13 - Daily Average Wind Speed for 2004

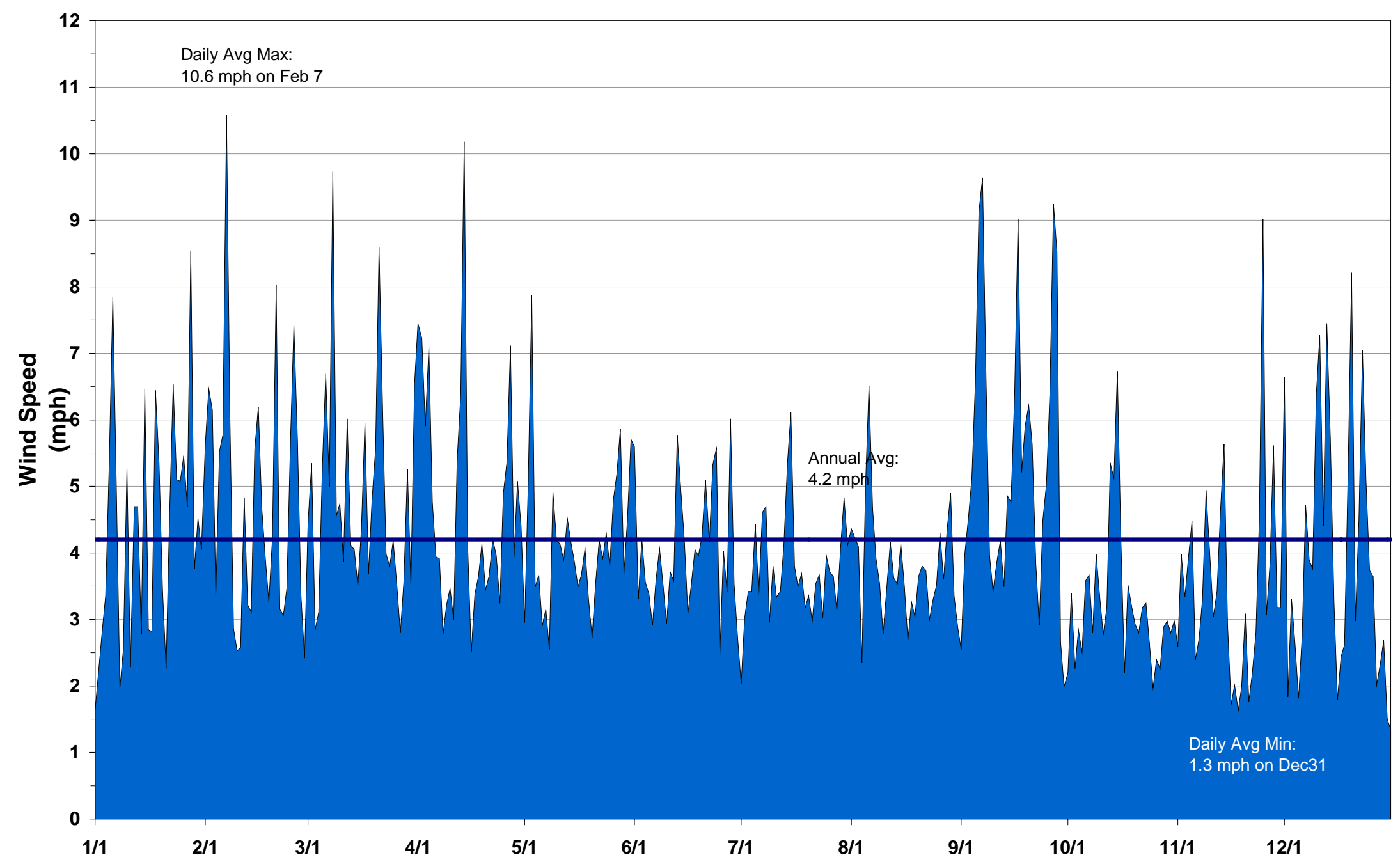


Fig. 14(a) - Annual Wind Rose Plots for 2004, 61-m Level

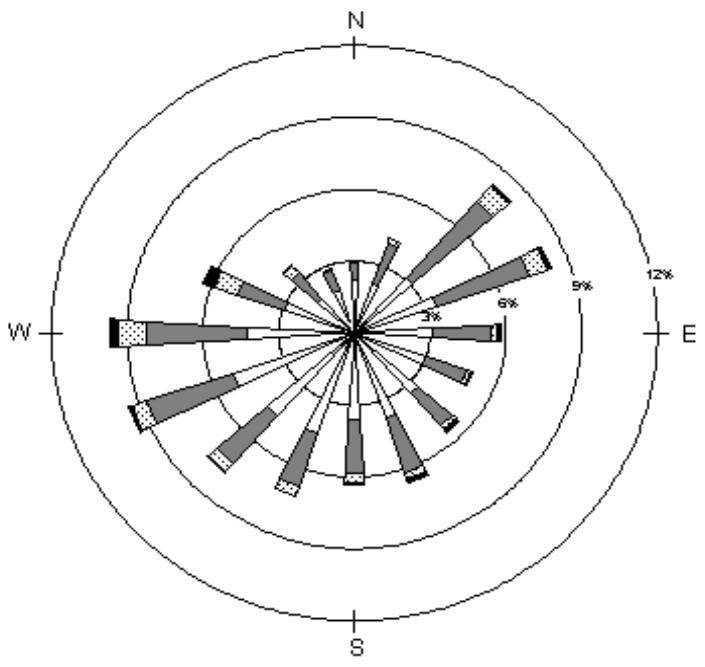

A Area Tower

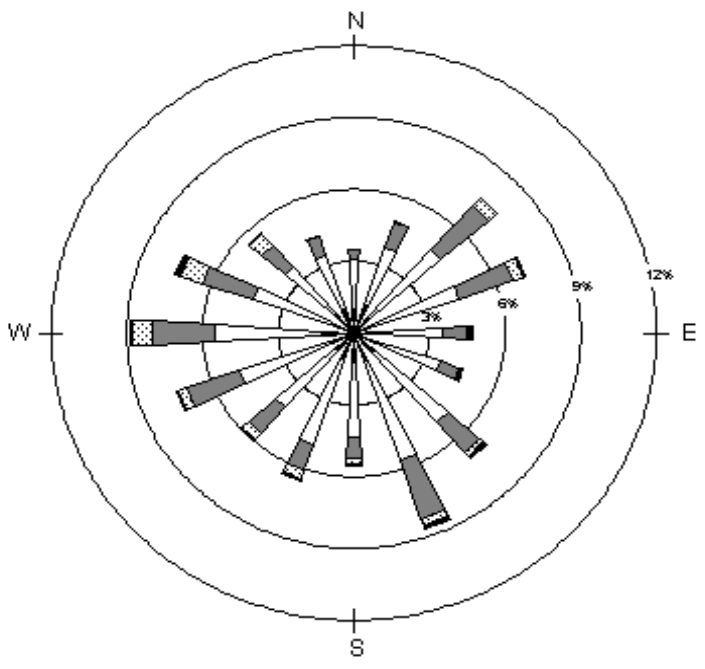

D Area Tower

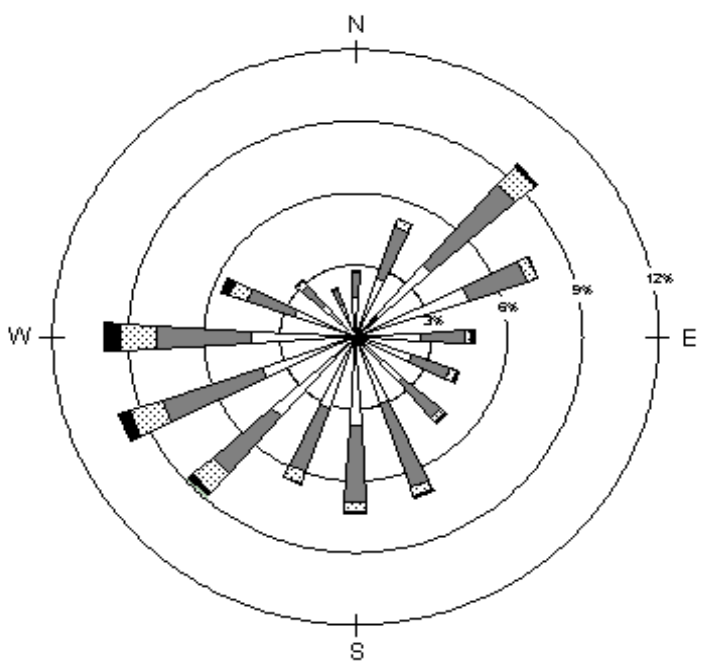

C Area Tower

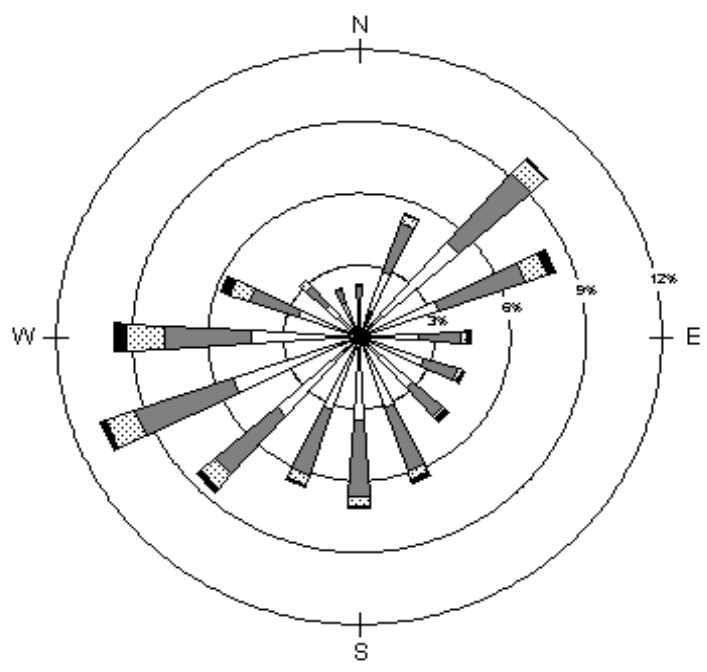

F Area Tower

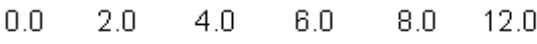 \\ Wind Speed Class Boundaries (Metersisecond)}

Wind rose plots depict the frequency of occurrence of wind direction sector (direction from wich the wind blows) by wind speed categoru 
Fig. 14(b) - Annual Wind Rose Plots for 2004, 61-m Level

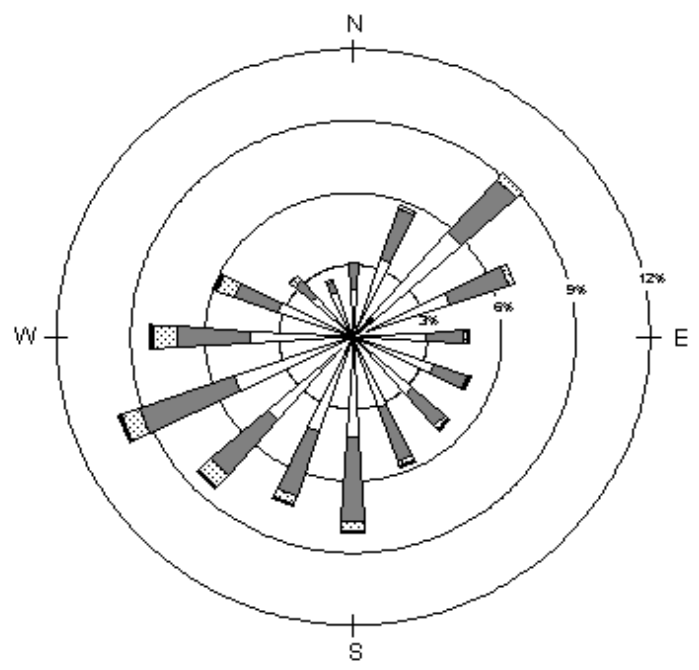

$\mathrm{H}$ Area Tower

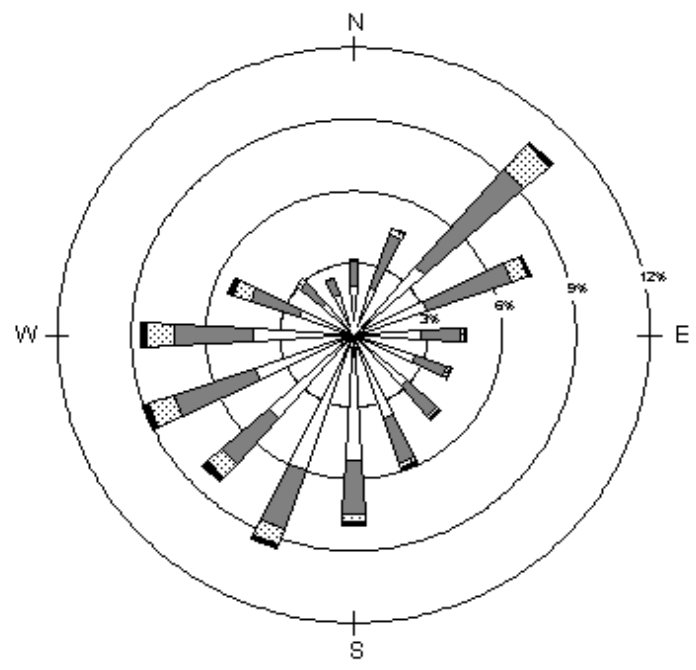

$\mathrm{L}$ Area Tower

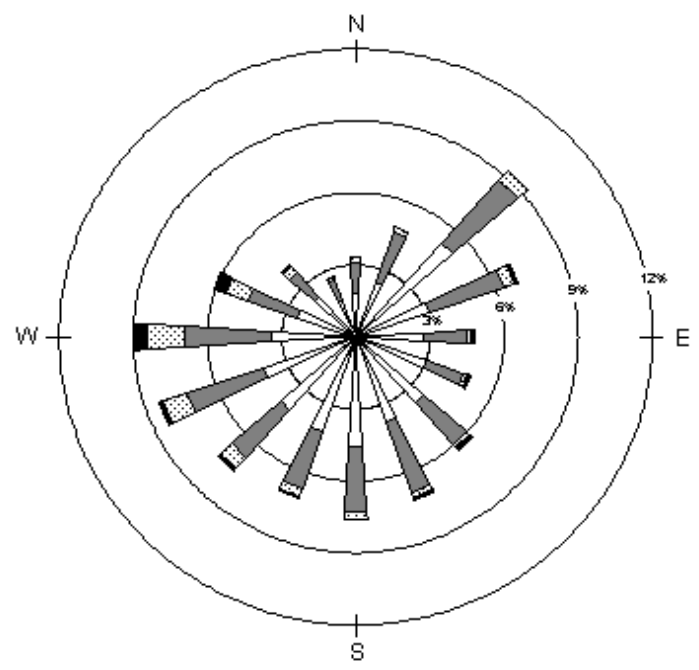

K Area Tower

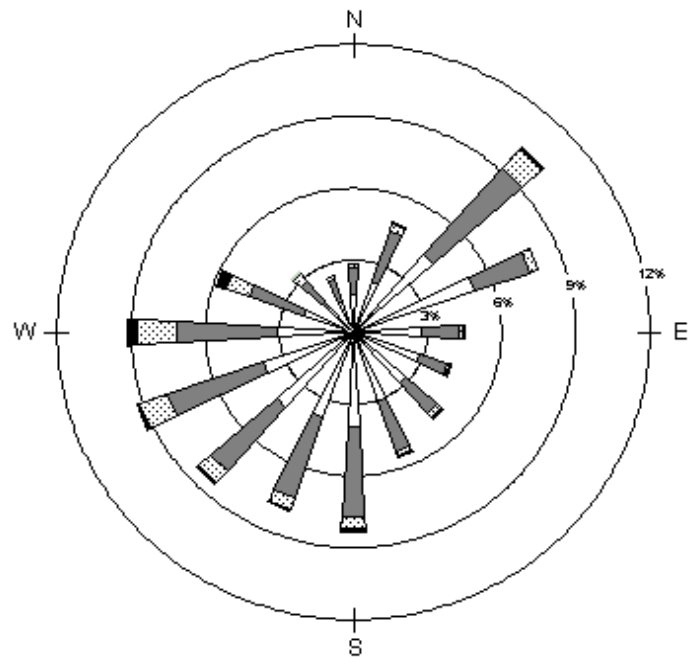

P Area Tower

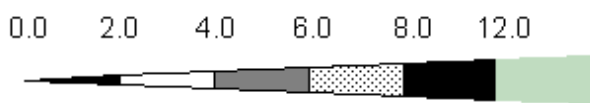

Wind Speed Class Boundaries (M etersisecond)

Wind rose plots depict the frequency of occurrence of wind direction sector (direction from wich the wind blows) by wind speed categoru 
Fig. 15 - Annual Wind Rose Plots for 2004, Central Climatology, All Levels

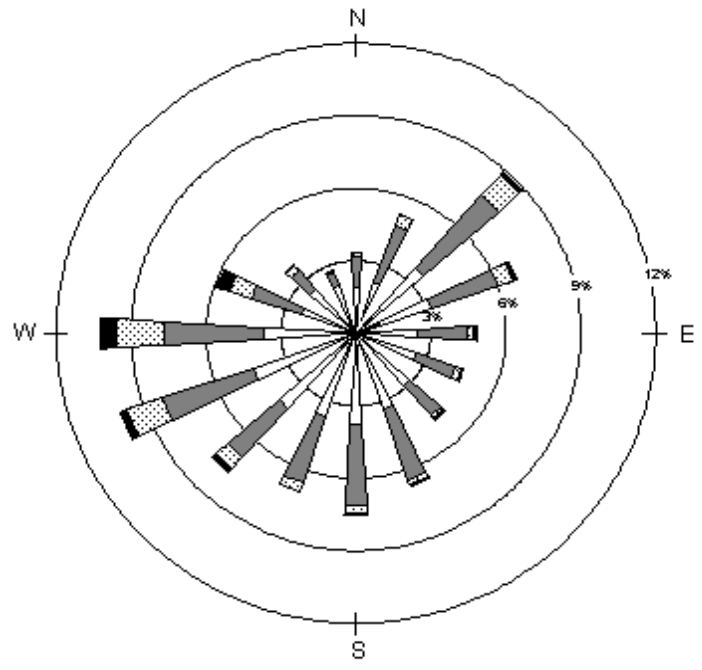

$61 \mathrm{~m}$ Level

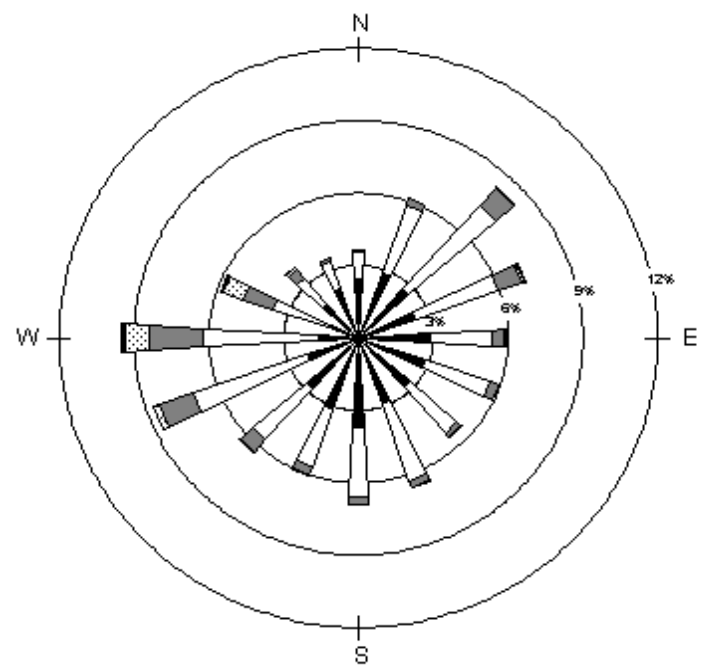

$18 \mathrm{~m}$ Level

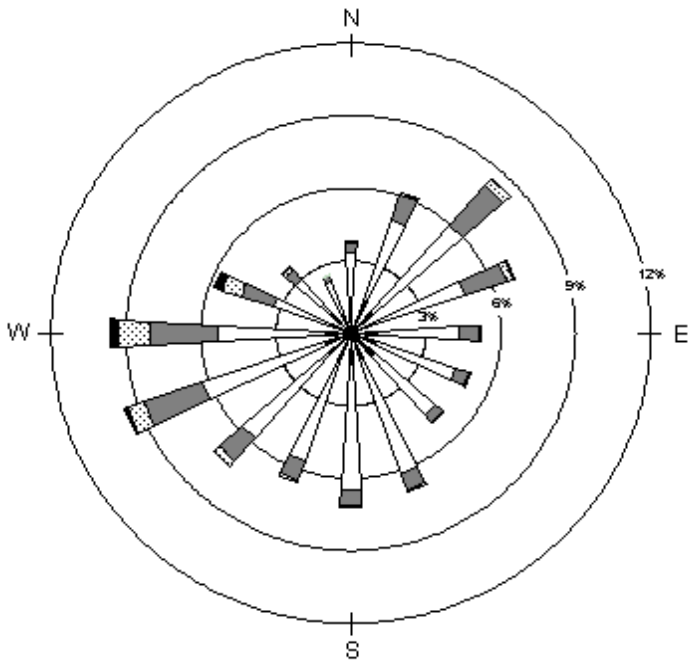

$36 \mathrm{~m}$ Level

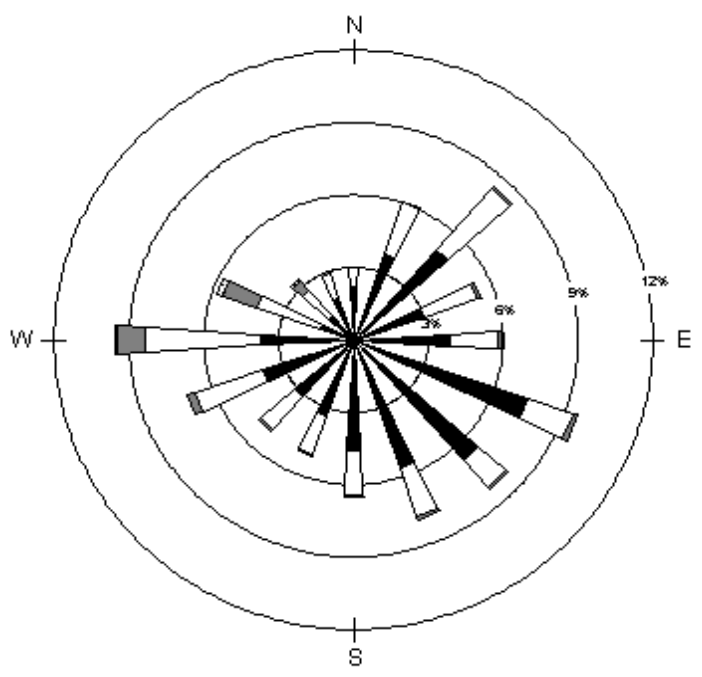

$4 \mathrm{~m}$ Level

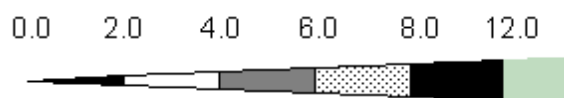

Wind Speed Class Boundaries (M etersisecond)

Wind rose plots depict the frequency of occurrence of wind direction sector (direction from wich the wind blows) by wind speed categoru 
Fig. 16 - Seasonal Wind Rose Plots for 2004, Central Climatology, 61-m Level

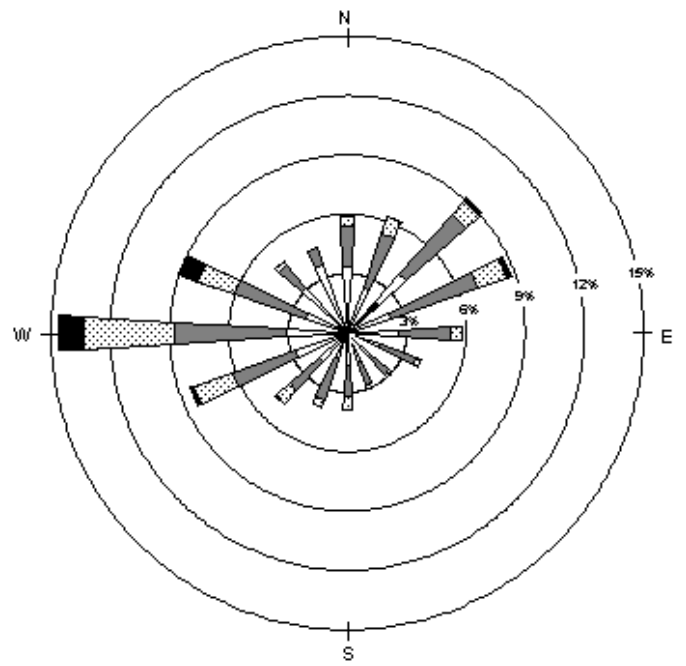

Winter

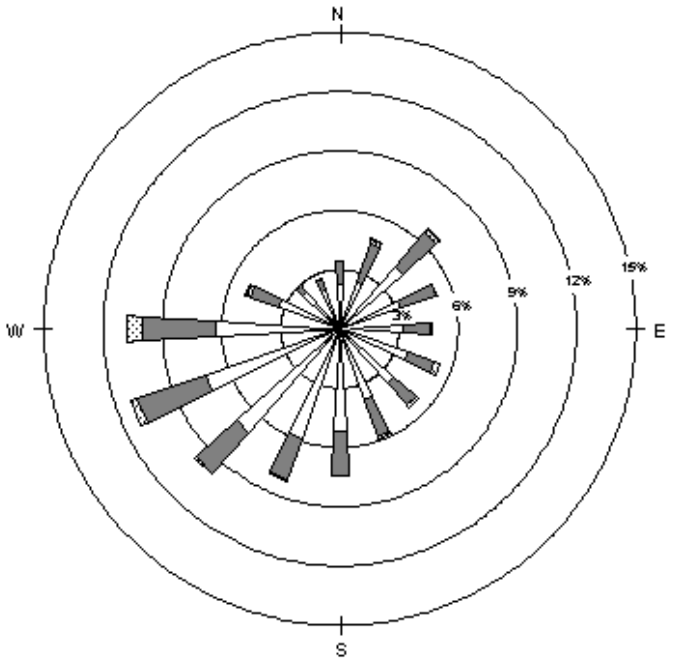

Summer

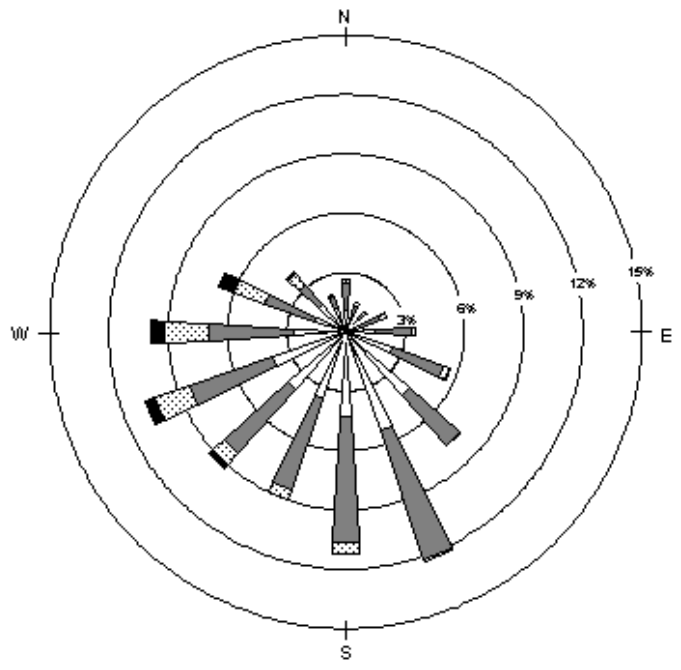

Spring

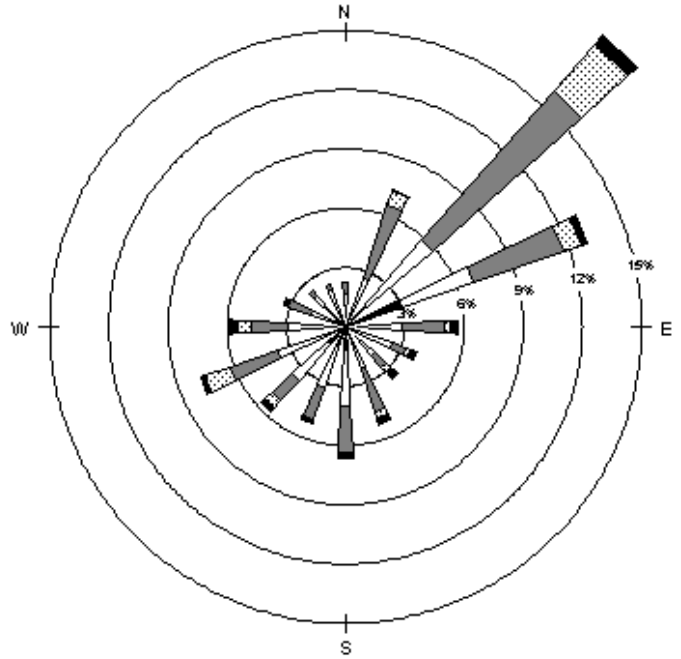

Fall

\section{$\begin{array}{llllll}0.0 & 2.0 & 4.0 & 6.0 & 8.0 & 12.0\end{array}$}

Wind Speed Class Boundaries

(M etersisecond)

Wind rose plots depict the frequency of occurrence of wind direction sector (direction from wich the wind blows) by wind speed categoru 
Fig. 17 - Daily Average Barometric Pressure for 2004

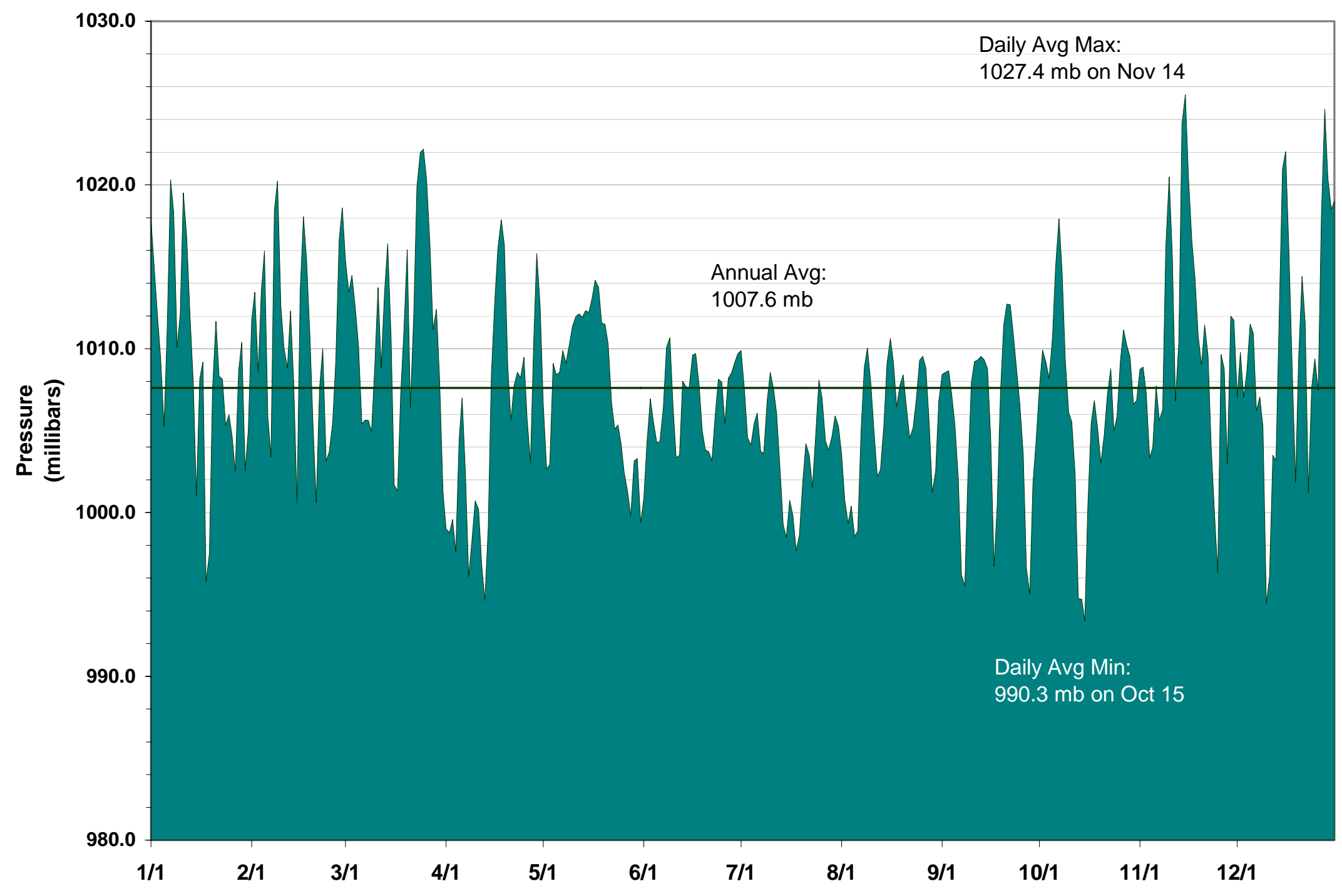


Fig. 18 - Daily Solar Radiation for 2004

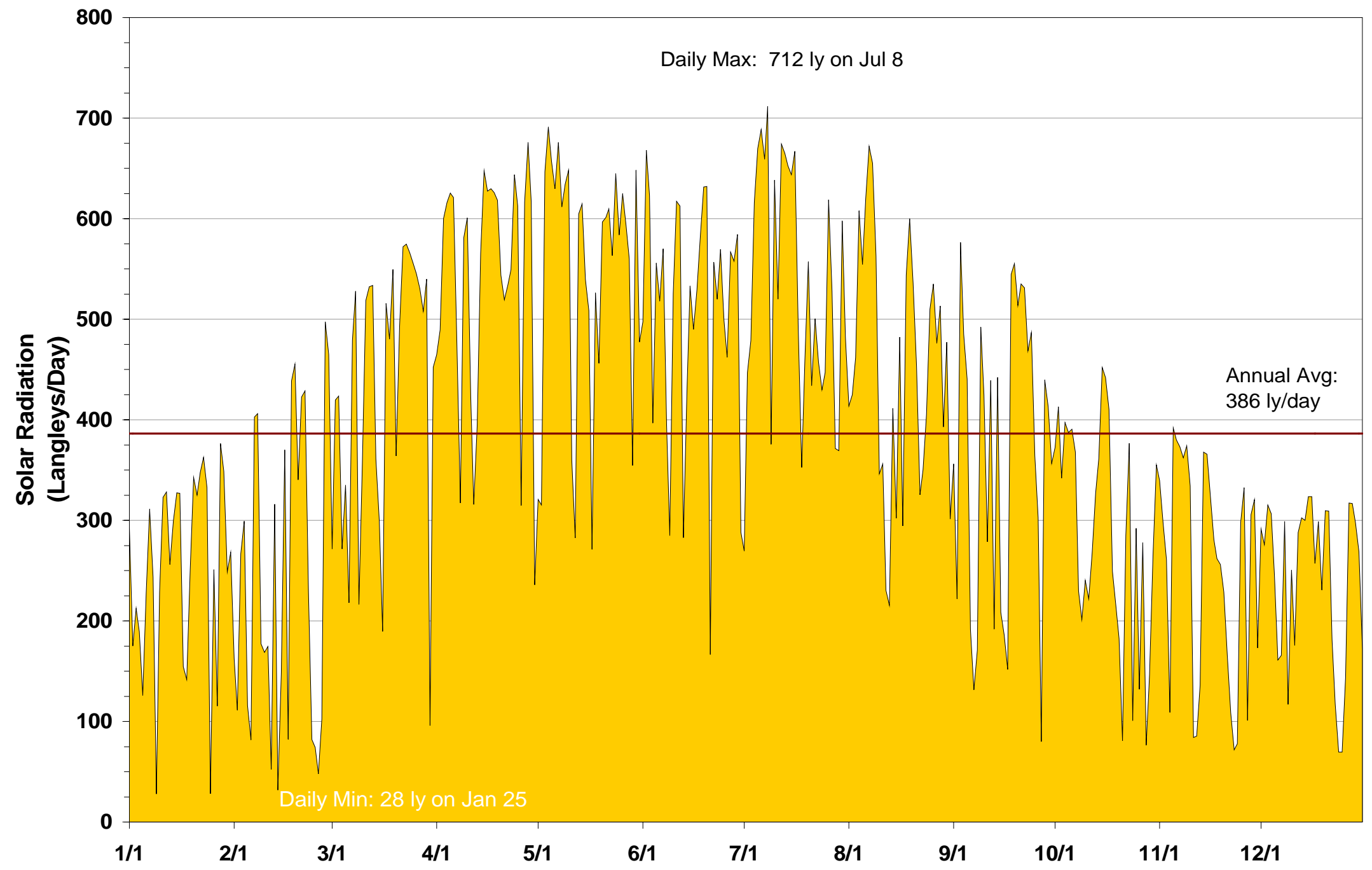


Table A.1 - Joint Occurrence Frequencies of Wind Direction Sector by Wind Speed Category for the A Area Tower, 2004

\begin{tabular}{|c|c|c|c|c|c|c|c|}
\hline \multirow[b]{2}{*}{ Sector } & \multicolumn{6}{|c|}{ Wind Speed Category, meters/sec } & \multirow[b]{2}{*}{ Total } \\
\hline & $0-2$ & 2-4 & 4-6 & 6-8 & 8-12 & $>12$ & \\
\hline $\mathbf{N}$ & 0.00638 & 0.01571 & 0.00754 & 0.00040 & 0.00000 & 0.00000 & 0.03003 \\
\hline NNE & 0.00515 & 0.01688 & 0.01788 & 0.00196 & 0.00006 & 0.00000 & 0.04193 \\
\hline $\mathrm{NE}$ & 0.00729 & 0.02374 & 0.04207 & 0.00854 & 0.00114 & 0.00000 & 0.08277 \\
\hline ENE & 0.00461 & 0.02966 & 0.03888 & 0.00774 & 0.00131 & 0.00000 & 0.08220 \\
\hline E & 0.00612 & 0.02522 & 0.02303 & 0.00222 & 0.00137 & 0.00000 & 0.05795 \\
\hline ESE & 0.00524 & 0.02516 & 0.01642 & 0.00199 & 0.00094 & 0.00003 & 0.04978 \\
\hline SE & 0.00606 & 0.02738 & 0.01819 & 0.00202 & 0.00188 & 0.00000 & 0.05553 \\
\hline SSE & 0.00720 & 0.03051 & 0.02388 & 0.00276 & 0.00188 & 0.00006 & 0.06629 \\
\hline$s$ & 0.00774 & 0.02866 & 0.02177 & 0.00396 & 0.00085 & 0.00003 & 0.06302 \\
\hline ssw & 0.00769 & 0.03649 & 0.02320 & 0.00438 & 0.00054 & 0.00000 & 0.07230 \\
\hline sw & 0.00800 & 0.03789 & 0.02718 & 0.00404 & 0.00071 & 0.00000 & 0.07782 \\
\hline wsw & 0.00780 & 0.04301 & 0.03598 & 0.00643 & 0.00111 & 0.00000 & 0.09433 \\
\hline wsw & 0.00757 & 0.03521 & 0.03945 & 0.01187 & 0.00282 & 0.00000 & 0.09692 \\
\hline WNW & 0.00734 & 0.02192 & 0.02004 & 0.00951 & 0.00430 & 0.00000 & 0.06310 \\
\hline NW & 0.00447 & 0.01480 & 0.01412 & 0.00370 & 0.00074 & 0.00006 & 0.03789 \\
\hline NNW & 0.00490 & 0.01392 & 0.00894 & 0.00037 & 0.00000 & 0.00003 & 0.02815 \\
\hline Total & 0.10355 & 0.42616 & 0.37856 & 0.07190 & 0.01964 & 0.00020 & 1.00000 \\
\hline
\end{tabular}

Table A.2 - Joint Occurrence Frequencies of Wind Direction Sector by Wind Speed Category for the C Area Tower, 2004

\begin{tabular}{|c|c|c|c|c|c|c|c|}
\hline \multirow[b]{2}{*}{ Sector } & \multicolumn{6}{|c|}{ Wind Speed Category, meters/sec } & \multirow[b]{2}{*}{ Total } \\
\hline & $0-2$ & $2-4$ & 4-6 & 6-8 & 8-12 & $>12$ & \\
\hline $\mathbf{N}$ & 0.00382 & 0.01248 & 0.01057 & 0.00060 & 0.00003 & 0.00000 & 0.02750 \\
\hline NNE & 0.00607 & 0.01975 & 0.02257 & 0.00336 & 0.00009 & 0.00003 & 0.05187 \\
\hline NE & 0.01211 & 0.02796 & 0.04489 & 0.01009 & 0.00117 & 0.00000 & 0.09622 \\
\hline ENE & 0.00644 & 0.04067 & 0.02445 & 0.00459 & 0.00034 & 0.00000 & 0.07650 \\
\hline E & 0.00485 & 0.02083 & 0.01807 & 0.00202 & 0.00148 & 0.00000 & 0.04725 \\
\hline ESE & 0.00416 & 0.01947 & 0.01599 & 0.00319 & 0.00088 & 0.00000 & 0.04369 \\
\hline SE & 0.00522 & 0.02009 & 0.01949 & 0.00228 & 0.00140 & 0.00000 & 0.04848 \\
\hline SSE & 0.00524 & 0.02460 & 0.03654 & 0.00356 & 0.00134 & 0.00000 & 0.07128 \\
\hline$s$ & 0.00650 & 0.03013 & 0.03195 & 0.00428 & 0.00094 & 0.00000 & 0.07379 \\
\hline ssw & 0.00630 & 0.02539 & 0.02790 & 0.00510 & 0.00068 & 0.00000 & 0.06538 \\
\hline sw & 0.00713 & 0.03719 & 0.03132 & 0.01069 & 0.00285 & 0.00009 & 0.08926 \\
\hline wsw & 0.00644 & 0.03266 & 0.04192 & 0.01337 & 0.00467 & 0.00003 & 0.09910 \\
\hline wsw & 0.00593 & 0.03583 & 0.03739 & 0.01397 & 0.00670 & 0.00003 & 0.09984 \\
\hline WNW & 0.00553 & 0.02009 & 0.01949 & 0.00778 & 0.00348 & 0.00003 & 0.05640 \\
\hline NW & 0.00416 & 0.01371 & 0.01086 & 0.00254 & 0.00040 & 0.00000 & 0.03167 \\
\hline NNW & 0.00311 & 0.01134 & 0.00661 & 0.00057 & 0.00014 & 0.00000 & 0.02178 \\
\hline Total & 0.09300 & 0.39220 & 0.40003 & 0.08798 & 0.02660 & 0.00020 & 1.00001 \\
\hline
\end{tabular}


Table A.3 - Joint Occurrence Frequencies of Wind Direction Sector by Wind Speed Category for the D Area Tower, 2004

\begin{tabular}{|c|c|c|c|c|c|c|c|}
\hline \multirow[b]{2}{*}{ Sector } & \multicolumn{6}{|c|}{ Wind Speed Category, meters/sec } & \multirow[b]{2}{*}{ Total } \\
\hline & $0-2$ & $2-4$ & $4-6$ & $6-8$ & $8-12$ & $>12$ & \\
\hline $\mathbf{N}$ & 0.01016 & 0.02083 & 0.00407 & 0.00009 & 0.00000 & 0.00000 & 0.03515 \\
\hline NNE & 0.01241 & 0.02516 & 0.01141 & 0.00048 & 0.00003 & 0.00000 & 0.04949 \\
\hline NE & 0.00882 & 0.03737 & 0.02499 & 0.00421 & 0.00026 & 0.00000 & 0.07565 \\
\hline ENE & 0.00848 & 0.03575 & 0.02260 & 0.00447 & 0.00043 & 0.00000 & 0.07172 \\
\hline$E$ & 0.00902 & 0.02633 & 0.00934 & 0.00128 & 0.00120 & 0.00000 & 0.04716 \\
\hline ESE & 0.00968 & 0.02633 & 0.00806 & 0.00097 & 0.00083 & 0.00000 & 0.04585 \\
\hline SE & 0.01139 & 0.03848 & 0.01662 & 0.00194 & 0.00171 & 0.00000 & 0.07013 \\
\hline SSE & 0.01241 & 0.04395 & 0.02567 & 0.00305 & 0.00174 & 0.00003 & 0.08684 \\
\hline$S$ & 0.01272 & 0.03074 & 0.00882 & 0.00239 & 0.00122 & 0.00000 & 0.05590 \\
\hline ssw & 0.01204 & 0.03692 & 0.01153 & 0.00404 & 0.00046 & 0.00000 & 0.06498 \\
\hline sw & 0.01013 & 0.03122 & 0.01415 & 0.00376 & 0.00074 & 0.00000 & 0.06000 \\
\hline WSw & 0.01010 & 0.03814 & 0.02166 & 0.00364 & 0.00083 & 0.00000 & 0.07437 \\
\hline wsw & 0.01264 & 0.04295 & 0.02459 & 0.00754 & 0.00120 & 0.00000 & 0.08891 \\
\hline WNW & 0.01016 & 0.03199 & 0.02155 & 0.00891 & 0.00219 & 0.00000 & 0.07480 \\
\hline NW & 0.01107 & 0.02536 & 0.01235 & 0.00646 & 0.00066 & 0.00006 & 0.05596 \\
\hline NNW & 0.01067 & 0.02382 & 0.00828 & 0.00023 & 0.00006 & 0.00003 & 0.04309 \\
\hline Total & 0.17191 & 0.51533 & 0.24568 & 0.05345 & 0.01352 & 0.00011 & 1.00000 \\
\hline
\end{tabular}

Table A.4 - Joint Occurrence Frequencies of Wind Direction Sector by Wind Speed Category for the F Area Tower, 2004

\begin{tabular}{|c|c|c|c|c|c|c|c|}
\hline \multirow[b]{2}{*}{ Sector } & \multicolumn{6}{|c|}{ Wind Speed Category, meters/sec } & \multirow[b]{2}{*}{ Total } \\
\hline & $0-2$ & $2-4$ & 4-6 & 6-8 & 8-12 & $>12$ & \\
\hline $\mathbf{N}$ & 0.00359 & 0.00846 & 0.00948 & 0.00068 & 0.00003 & 0.00000 & 0.02224 \\
\hline NNE & 0.01110 & 0.01765 & 0.02130 & 0.00421 & 0.00011 & 0.00000 & 0.05438 \\
\hline NE & 0.00766 & 0.04519 & 0.03812 & 0.00812 & 0.00091 & 0.00000 & 0.09999 \\
\hline ENE & 0.00604 & 0.02708 & 0.03636 & 0.00945 & 0.00339 & 0.00006 & 0.08237 \\
\hline$E$ & 0.00507 & 0.01874 & 0.01632 & 0.00231 & 0.00165 & 0.00000 & 0.04408 \\
\hline ESE & 0.00655 & 0.02070 & 0.01421 & 0.00165 & 0.00103 & 0.00000 & 0.04413 \\
\hline SE & 0.00547 & 0.02261 & 0.01563 & 0.00177 & 0.00174 & 0.00000 & 0.04721 \\
\hline SSE & 0.00564 & 0.02543 & 0.02827 & 0.00310 & 0.00171 & 0.00000 & 0.06415 \\
\hline$s$ & 0.00715 & 0.02790 & 0.03158 & 0.00396 & 0.00094 & 0.00000 & 0.07152 \\
\hline ssw & 0.00681 & 0.02580 & 0.02819 & 0.00458 & 0.00071 & 0.00003 & 0.06611 \\
\hline sw & 0.00886 & 0.03485 & 0.03249 & 0.00797 & 0.00222 & 0.00000 & 0.08639 \\
\hline WSw & 0.00883 & 0.04390 & 0.04126 & 0.01219 & 0.00216 & 0.00000 & 0.10834 \\
\hline WSW & 0.00783 & 0.03542 & 0.03420 & 0.01472 & 0.00510 & 0.00000 & 0.09726 \\
\hline WNW & 0.00658 & 0.01902 & 0.02044 & 0.00812 & 0.00387 & 0.00006 & 0.05808 \\
\hline NW & 0.00470 & 0.01333 & 0.01065 & 0.00293 & 0.00037 & 0.00000 & 0.03198 \\
\hline NNW & 0.00362 & 0.01159 & 0.00615 & 0.00031 & 0.00009 & 0.00003 & 0.02178 \\
\hline Total & 0.10546 & 0.39764 & 0.38463 & 0.08607 & 0.02602 & 0.00017 & 1.00000 \\
\hline
\end{tabular}


Table A.5 - Joint Occurrence Frequencies of Wind Direction Sector by Wind Speed Category for the H Area Tower, 2004

\begin{tabular}{|c|c|c|c|c|c|c|c|}
\hline \multirow[b]{2}{*}{ Sector } & \multicolumn{6}{|c|}{ Wind Speed Category, meters/sec } & \multirow[b]{2}{*}{ Total } \\
\hline & $0-2$ & $2-4$ & $4-6$ & $6-8$ & $8-12$ & $>12$ & \\
\hline $\mathbf{N}$ & 0.00586 & 0.01395 & 0.01087 & 0.00046 & 0.00000 & 0.00003 & 0.03116 \\
\hline NNE & 0.00860 & 0.02570 & 0.02194 & 0.00159 & 0.00006 & 0.00000 & 0.05789 \\
\hline NE & 0.01116 & 0.04696 & 0.02954 & 0.00393 & 0.00017 & 0.00003 & 0.09179 \\
\hline ENE & 0.00589 & 0.03526 & 0.02496 & 0.00253 & 0.00020 & 0.00000 & 0.06885 \\
\hline$E$ & 0.00535 & 0.02414 & 0.01514 & 0.00182 & 0.00043 & 0.00000 & 0.04688 \\
\hline ESE & 0.00595 & 0.02838 & 0.01429 & 0.00094 & 0.00051 & 0.00000 & 0.05006 \\
\hline SE & 0.00572 & 0.02636 & 0.01728 & 0.00185 & 0.00094 & 0.00000 & 0.05214 \\
\hline SSE & 0.00552 & 0.02564 & 0.02348 & 0.00222 & 0.00100 & 0.00000 & 0.05786 \\
\hline$S$ & 0.00766 & 0.03421 & 0.03484 & 0.00441 & 0.00063 & 0.00000 & 0.08174 \\
\hline ssw & 0.00743 & 0.03430 & 0.02840 & 0.00367 & 0.00066 & 0.00000 & 0.07445 \\
\hline sw & 0.00712 & 0.03845 & 0.03111 & 0.00626 & 0.00114 & 0.00000 & 0.08407 \\
\hline WSw & 0.00583 & 0.04483 & 0.04093 & 0.00768 & 0.00137 & 0.00000 & 0.10064 \\
\hline wsw & 0.00578 & 0.03578 & 0.02977 & 0.00982 & 0.00171 & 0.00000 & 0.08285 \\
\hline WNW & 0.00677 & 0.02579 & 0.01804 & 0.00763 & 0.00179 & 0.00000 & 0.06003 \\
\hline NW & 0.00555 & 0.01699 & 0.00879 & 0.00256 & 0.00034 & 0.00000 & 0.03424 \\
\hline NNW & 0.00527 & 0.01452 & 0.00484 & 0.00068 & 0.00003 & 0.00003 & 0.02536 \\
\hline Total & 0.10545 & 0.47123 & 0.35422 & 0.05806 & 0.01096 & 0.00008 & 0.99999 \\
\hline
\end{tabular}

Table A.6 - Joint Occurrence Frequencies of Wind Direction Sector by Wind Speed Category for the K Area Tower, 2004

\begin{tabular}{|c|c|c|c|c|c|c|c|}
\hline \multirow[b]{2}{*}{ Sector } & \multicolumn{6}{|c|}{ Wind Speed Category, meters/sec } & \multirow[b]{2}{*}{ Total } \\
\hline & $0-2$ & $2-4$ & 4-6 & 6-8 & 8-12 & $>12$ & \\
\hline $\mathbf{N}$ & 0.00404 & 0.01403 & 0.01326 & 0.00174 & 0.00003 & 0.00000 & 0.03310 \\
\hline NNE & 0.00578 & 0.01839 & 0.02214 & 0.00202 & 0.00009 & 0.00000 & 0.04841 \\
\hline NE & 0.00612 & 0.04605 & 0.03546 & 0.00495 & 0.00023 & 0.00000 & 0.09281 \\
\hline ENE & 0.00444 & 0.02718 & 0.03162 & 0.00487 & 0.00100 & 0.00000 & 0.06911 \\
\hline$E$ & 0.00458 & 0.02314 & 0.01736 & 0.00179 & 0.00140 & 0.00000 & 0.04827 \\
\hline ESE & 0.00649 & 0.02399 & 0.01560 & 0.00168 & 0.00114 & 0.00000 & 0.04890 \\
\hline SE & 0.00626 & 0.02991 & 0.02328 & 0.00168 & 0.00168 & 0.00000 & 0.06282 \\
\hline SSE & 0.00714 & 0.03031 & 0.03102 & 0.00225 & 0.00140 & 0.00000 & 0.07212 \\
\hline$s$ & 0.00840 & 0.03709 & 0.02744 & 0.00290 & 0.00051 & 0.00000 & 0.07633 \\
\hline ssw & 0.00786 & 0.03381 & 0.02490 & 0.00410 & 0.00057 & 0.00000 & 0.07124 \\
\hline sw & 0.00692 & 0.03344 & 0.02653 & 0.00635 & 0.00134 & 0.00000 & 0.07457 \\
\hline WSw & 0.00652 & 0.03307 & 0.03248 & 0.01065 & 0.00151 & 0.00000 & 0.08422 \\
\hline WSW & 0.00595 & 0.02818 & 0.03515 & 0.01528 & 0.00581 & 0.00003 & 0.09039 \\
\hline WNW & 0.00535 & 0.01955 & 0.02103 & 0.00968 & 0.00501 & 0.00014 & 0.06077 \\
\hline NW & 0.00470 & 0.01736 & 0.01380 & 0.00370 & 0.00083 & 0.00003 & 0.04042 \\
\hline NNW & 0.00404 & 0.01201 & 0.00925 & 0.00097 & 0.00020 & 0.00006 & 0.02653 \\
\hline Total & 0.09458 & 0.42752 & 0.38033 & 0.07460 & 0.02271 & 0.00026 & 1.00000 \\
\hline
\end{tabular}


Table A.7 - Joint Occurrence Frequencies of Wind Direction Sector by Wind Speed Category for the L Area Tower, 2004

\begin{tabular}{|c|c|c|c|c|c|c|c|}
\hline \multirow[b]{2}{*}{ Sector } & \multicolumn{6}{|c|}{ Wind Speed Category, meters/sec } & \multirow[b]{2}{*}{ Total } \\
\hline & $0-2$ & $2-4$ & $4-6$ & $6-8$ & $8-12$ & $>12$ & \\
\hline$N$ & 0.00589 & 0.01452 & 0.01047 & 0.00103 & 0.00000 & 0.00000 & 0.03191 \\
\hline NNE & 0.00379 & 0.01676 & 0.02345 & 0.00310 & 0.00009 & 0.00000 & 0.04719 \\
\hline NE & 0.00945 & 0.02846 & 0.05399 & 0.01398 & 0.00174 & 0.00000 & 0.10762 \\
\hline ENE & 0.00470 & 0.02684 & 0.03612 & 0.00692 & 0.00111 & 0.00000 & 0.07568 \\
\hline E & 0.00524 & 0.02237 & 0.01580 & 0.00157 & 0.00054 & 0.00000 & 0.04551 \\
\hline ESE & 0.00581 & 0.02069 & 0.01324 & 0.00165 & 0.00063 & 0.00000 & 0.04201 \\
\hline SE & 0.00595 & 0.02240 & 0.01659 & 0.00122 & 0.00117 & 0.00000 & 0.04733 \\
\hline SSE & 0.00584 & 0.03054 & 0.02038 & 0.00165 & 0.00114 & 0.00000 & 0.05954 \\
\hline s & 0.00894 & 0.04363 & 0.02234 & 0.00336 & 0.00091 & 0.00000 & 0.07918 \\
\hline ssw & 0.00902 & 0.05046 & 0.02607 & 0.00712 & 0.00179 & 0.00003 & 0.09450 \\
\hline sw & 0.00697 & 0.03931 & 0.02519 & 0.00751 & 0.00290 & 0.00003 & 0.08191 \\
\hline wsw & 0.00638 & 0.03578 & 0.03495 & 0.01090 & 0.00256 & 0.00000 & 0.09057 \\
\hline wsw & 0.00623 & 0.03447 & 0.03208 & 0.01139 & 0.00265 & 0.00000 & 0.08681 \\
\hline WNW & 0.00487 & 0.01867 & 0.02072 & 0.00717 & 0.00228 & 0.00000 & 0.05371 \\
\hline NW & 0.00381 & 0.01358 & 0.01150 & 0.00219 & 0.00029 & 0.00003 & 0.03140 \\
\hline NNW & 0.00427 & 0.01358 & 0.00677 & 0.00048 & 0.00003 & 0.00000 & 0.02513 \\
\hline Total & 0.09714 & 0.43206 & 0.36967 & 0.08123 & 0.01981 & 0.00008 & 1.00000 \\
\hline
\end{tabular}

Table A.8 - Joint Occurrence Frequencies of Wind Direction Sector by Wind Speed Category for the P Area Tower, 2004

\begin{tabular}{|c|c|c|c|c|c|c|c|}
\hline \multirow[b]{2}{*}{ Sector } & \multicolumn{6}{|c|}{ Wind Speed Category, meters/sec } & \multirow[b]{2}{*}{ Total } \\
\hline & $0-2$ & $2-4$ & 4-6 & 6-8 & 8-12 & $>12$ & \\
\hline $\mathbf{N}$ & 0.00427 & 0.01105 & 0.01196 & 0.00131 & 0.00003 & 0.00000 & 0.02861 \\
\hline NNE & 0.00575 & 0.01654 & 0.02252 & 0.00319 & 0.00026 & 0.00000 & 0.04825 \\
\hline NE & 0.01099 & 0.03194 & 0.04823 & 0.01070 & 0.00137 & 0.00000 & 0.10323 \\
\hline ENE & 0.00646 & 0.04578 & 0.02366 & 0.00316 & 0.00020 & 0.00000 & 0.07926 \\
\hline$E$ & 0.00575 & 0.02184 & 0.01480 & 0.00191 & 0.00048 & 0.00000 & 0.04478 \\
\hline ESE & 0.00606 & 0.02243 & 0.01164 & 0.00131 & 0.00068 & 0.00000 & 0.04213 \\
\hline SE & 0.00643 & 0.02186 & 0.01674 & 0.00216 & 0.00137 & 0.00000 & 0.04857 \\
\hline SSE & 0.00658 & 0.02403 & 0.02189 & 0.00188 & 0.00114 & 0.00000 & 0.05551 \\
\hline$s$ & 0.00752 & 0.03180 & 0.03803 & 0.00495 & 0.00137 & 0.00000 & 0.08367 \\
\hline ssw & 0.00686 & 0.03083 & 0.03567 & 0.00478 & 0.00085 & 0.00000 & 0.07900 \\
\hline sw & 0.00575 & 0.03587 & 0.03550 & 0.00626 & 0.00157 & 0.00000 & 0.08495 \\
\hline WSw & 0.00564 & 0.03280 & 0.04136 & 0.01176 & 0.00142 & 0.00000 & 0.09298 \\
\hline wsw & 0.00487 & 0.02582 & 0.04108 & 0.01569 & 0.00458 & 0.00003 & 0.09207 \\
\hline WNW & 0.00376 & 0.01742 & 0.02337 & 0.00974 & 0.00473 & 0.00003 & 0.05904 \\
\hline NW & 0.00327 & 0.01059 & 0.01423 & 0.00404 & 0.00097 & 0.00006 & 0.03317 \\
\hline NNW & 0.00293 & 0.01019 & 0.01033 & 0.00117 & 0.00011 & 0.00006 & 0.02480 \\
\hline Total & 0.09289 & 0.39078 & 0.41102 & 0.08401 & 0.02112 & 0.00017 & 1.00000 \\
\hline
\end{tabular}


Table A.9 - Joint Occurrence Frequencies of Wind Direction Sector by Wind Speed Category for the 4m Level Central Climatology Tower, 2004

\begin{tabular}{|c|c|c|c|c|c|c|c|}
\hline \multirow[b]{2}{*}{ Sector } & \multicolumn{6}{|c|}{ Wind Speed Category, meters/sec } & \multirow[b]{2}{*}{ Total } \\
\hline & $0-2$ & $2-4$ & 4-6 & $6-8$ & $8-12$ & $>12$ & \\
\hline$N$ & 0.02201 & 0.00771 & 0.00011 & 0.00000 & 0.00000 & 0.00000 & 0.02983 \\
\hline NNE & 0.03795 & 0.02218 & 0.00048 & 0.00000 & 0.00000 & 0.00000 & 0.06061 \\
\hline NE & 0.05013 & 0.03322 & 0.00171 & 0.00000 & 0.00000 & 0.00000 & 0.08506 \\
\hline ENE & 0.02955 & 0.02280 & 0.00179 & 0.00000 & 0.00000 & 0.00000 & 0.05414 \\
\hline$E$ & 0.03846 & 0.01922 & 0.00174 & 0.00006 & 0.00000 & 0.00000 & 0.05947 \\
\hline ESE & 0.07393 & 0.01924 & 0.00208 & 0.00014 & 0.00000 & 0.00000 & 0.09539 \\
\hline SE & 0.06701 & 0.01460 & 0.00122 & 0.00000 & 0.00000 & 0.00000 & 0.08284 \\
\hline SSE & 0.05634 & 0.02124 & 0.00108 & 0.00000 & 0.00000 & 0.00000 & 0.07865 \\
\hline S & 0.04606 & 0.01819 & 0.00077 & 0.00003 & 0.00000 & 0.00000 & 0.06505 \\
\hline ssw & 0.03359 & 0.01654 & 0.00071 & 0.00000 & 0.00000 & 0.00000 & 0.05084 \\
\hline sw & 0.03117 & 0.01802 & 0.00122 & 0.00000 & 0.00000 & 0.00000 & 0.05041 \\
\hline WSW & 0.03812 & 0.02943 & 0.00330 & 0.00000 & 0.00000 & 0.00000 & 0.07085 \\
\hline WSW & 0.03760 & 0.04577 & 0.01190 & 0.00040 & 0.00000 & 0.00000 & 0.09568 \\
\hline WNW & 0.01358 & 0.02716 & 0.01443 & 0.00279 & 0.00003 & 0.00000 & 0.05799 \\
\hline NW & 0.01329 & 0.01398 & 0.00532 & 0.00077 & 0.00000 & 0.00000 & 0.03336 \\
\hline NNW & 0.01859 & 0.01031 & 0.00080 & 0.00009 & 0.00006 & 0.00000 & 0.02983 \\
\hline Total & 0.60736 & 0.33960 & 0.04868 & 0.00427 & 0.00009 & 0.00000 & 1.00000 \\
\hline
\end{tabular}

Table A.10 - Joint Occurrence Frequencies of Wind Direction Sector by Wind Speed Category for the 18m Level Central Climatology Tower, 2004

\begin{tabular}{|c|c|c|c|c|c|c|c|}
\hline \multirow[b]{2}{*}{ Sector } & \multicolumn{6}{|c|}{ Wind Speed Category, meters/sec } & \multirow[b]{2}{*}{ Total } \\
\hline & $0-2$ & $2-4$ & 4-6 & $6-8$ & 8-12 & $>12$ & \\
\hline $\mathbf{N}$ & 0.02477 & 0.01079 & 0.00051 & 0.00003 & 0.00000 & 0.00000 & 0.03610 \\
\hline NNE & 0.02850 & 0.02946 & 0.00296 & 0.00003 & 0.00000 & 0.00000 & 0.06095 \\
\hline NE & 0.02702 & 0.04623 & 0.00982 & 0.00048 & 0.00000 & 0.00000 & 0.08355 \\
\hline ENE & 0.02408 & 0.03524 & 0.01005 & 0.00125 & 0.00006 & 0.00000 & 0.07068 \\
\hline E & 0.02878 & 0.02471 & 0.00501 & 0.00091 & 0.00009 & 0.00000 & 0.05950 \\
\hline ESE & 0.02841 & 0.02776 & 0.00353 & 0.00057 & 0.00009 & 0.00000 & 0.06035 \\
\hline SE & 0.02758 & 0.02491 & 0.00222 & 0.00043 & 0.00000 & 0.00000 & 0.05514 \\
\hline SSE & 0.02872 & 0.03373 & 0.00296 & 0.00031 & 0.00000 & 0.00000 & 0.06573 \\
\hline$S$ & 0.03704 & 0.02892 & 0.00288 & 0.00009 & 0.00000 & 0.00000 & 0.06892 \\
\hline ssw & 0.03157 & 0.02497 & 0.00367 & 0.00017 & 0.00000 & 0.00000 & 0.06038 \\
\hline sw & 0.02781 & 0.02844 & 0.00666 & 0.00068 & 0.00000 & 0.00000 & 0.06359 \\
\hline WSW & 0.02158 & 0.04868 & 0.01418 & 0.00262 & 0.00023 & 0.00000 & 0.08728 \\
\hline wsw & 0.01623 & 0.04626 & 0.02155 & 0.00942 & 0.00140 & 0.00000 & 0.09485 \\
\hline WNW & 0.01287 & 0.02320 & 0.01264 & 0.00834 & 0.00162 & 0.00000 & 0.05867 \\
\hline NW & 0.01907 & 0.01366 & 0.00484 & 0.00134 & 0.00006 & 0.00000 & 0.03897 \\
\hline NNW & 0.02175 & 0.01204 & 0.00142 & 0.00006 & 0.00009 & 0.00000 & 0.03535 \\
\hline Total & 0.40576 & 0.45900 & 0.10490 & 0.02673 & 0.00362 & 0.00000 & 1.00000 \\
\hline
\end{tabular}


Table A.11 - Joint Occurrence Frequencies of Wind Direction Sector by Wind Speed Category for the 36m Level Central Climatology Tower, 2004

\begin{tabular}{|c|c|c|c|c|c|c|c|}
\hline \multirow[b]{2}{*}{ Sector } & \multicolumn{6}{|c|}{ Wind Speed Category, meters/sec } & \multirow[b]{2}{*}{ Total } \\
\hline & $0-2$ & $2-4$ & $4-6$ & $6-8$ & $8-12$ & $>12$ & \\
\hline $\mathbf{N}$ & 0.00951 & 0.02351 & 0.00473 & 0.00011 & 0.00003 & 0.00000 & 0.03789 \\
\hline NNE & 0.01443 & 0.03476 & 0.01142 & 0.00083 & 0.00006 & 0.00000 & 0.06149 \\
\hline NE & 0.01207 & 0.04771 & 0.02161 & 0.00376 & 0.00014 & 0.00000 & 0.08529 \\
\hline ENE & 0.01050 & 0.03746 & 0.01768 & 0.00310 & 0.00031 & 0.00000 & 0.06906 \\
\hline$E$ & 0.01093 & 0.03240 & 0.00777 & 0.00111 & 0.00029 & 0.00000 & 0.05249 \\
\hline ESE & 0.01119 & 0.03305 & 0.00567 & 0.00046 & 0.00043 & 0.00000 & 0.05078 \\
\hline SE & 0.01273 & 0.03234 & 0.00376 & 0.00060 & 0.00003 & 0.00000 & 0.04945 \\
\hline SSE & 0.01156 & 0.04990 & 0.00703 & 0.00105 & 0.00000 & 0.00000 & 0.06954 \\
\hline S & 0.01332 & 0.05158 & 0.00669 & 0.00083 & 0.00006 & 0.00000 & 0.07248 \\
\hline ssw & 0.01304 & 0.04321 & 0.00823 & 0.00103 & 0.00006 & 0.00000 & 0.06556 \\
\hline sw & 0.01241 & 0.04543 & 0.01250 & 0.00299 & 0.00026 & 0.00000 & 0.07359 \\
\hline WSW & 0.01082 & 0.05218 & 0.02445 & 0.00709 & 0.00137 & 0.00000 & 0.09590 \\
\hline WSW & 0.00999 & 0.04373 & 0.02719 & 0.01230 & 0.00347 & 0.00000 & 0.09667 \\
\hline WNW & 0.00865 & 0.02403 & 0.01378 & 0.00800 & 0.00316 & 0.00000 & 0.05762 \\
\hline NW & 0.01122 & 0.01813 & 0.00535 & 0.00177 & 0.00020 & 0.00000 & 0.03667 \\
\hline NNW & 0.00900 & 0.01378 & 0.00256 & 0.00011 & 0.00006 & 0.00003 & 0.02553 \\
\hline Total & 0.18136 & 0.58320 & 0.18039 & 0.04512 & 0.00991 & 0.00003 & 1.00000 \\
\hline
\end{tabular}

Table A.12 - Joint Occurrence Frequencies of Wind Direction Sector by Wind Speed Category for the 61m Level Central Climatology Tower, 2004

\begin{tabular}{|c|c|c|c|c|c|c|c|}
\hline \multirow[b]{2}{*}{ Sector } & \multicolumn{6}{|c|}{ Wind Speed Category, meters/sec } & \multirow[b]{2}{*}{ Total } \\
\hline & $0-2$ & $2-4$ & $4-6$ & $6-8$ & 8-12 & $>12$ & \\
\hline $\mathbf{N}$ & 0.00501 & 0.01389 & 0.01298 & 0.00117 & 0.00003 & 0.00003 & 0.03311 \\
\hline NNE & 0.00595 & 0.01637 & 0.02494 & 0.00493 & 0.00023 & 0.00000 & 0.05241 \\
\hline NE & 0.00957 & 0.02605 & 0.04057 & 0.01093 & 0.00219 & 0.00000 & 0.08930 \\
\hline ENE & 0.01016 & 0.02189 & 0.02832 & 0.00606 & 0.00140 & 0.00000 & 0.06784 \\
\hline$E$ & 0.00475 & 0.02015 & 0.01978 & 0.00265 & 0.00122 & 0.00000 & 0.04856 \\
\hline ESE & 0.00567 & 0.02021 & 0.01645 & 0.00253 & 0.00085 & 0.00000 & 0.04572 \\
\hline SE & 0.00712 & 0.02149 & 0.01688 & 0.00162 & 0.00111 & 0.00000 & 0.04822 \\
\hline SSE & 0.00900 & 0.02434 & 0.03060 & 0.00216 & 0.00091 & 0.00000 & 0.06701 \\
\hline $\mathbf{s}$ & 0.00808 & 0.02986 & 0.03322 & 0.00319 & 0.00066 & 0.00000 & 0.07501 \\
\hline ssw & 0.00700 & 0.02929 & 0.02915 & 0.00384 & 0.00043 & 0.00000 & 0.06972 \\
\hline sw & 0.00874 & 0.03251 & 0.02761 & 0.00646 & 0.00171 & 0.00000 & 0.07703 \\
\hline WSW & 0.00763 & 0.03581 & 0.03869 & 0.01495 & 0.00273 & 0.00000 & 0.09980 \\
\hline WSW & 0.00530 & 0.03171 & 0.04000 & 0.01924 & 0.00601 & 0.00000 & 0.10225 \\
\hline WNW & 0.00578 & 0.01734 & 0.02115 & 0.00925 & 0.00535 & 0.00000 & 0.05887 \\
\hline NW & 0.00666 & 0.01762 & 0.01019 & 0.00299 & 0.00040 & 0.00000 & 0.03786 \\
\hline NNW & 0.00524 & 0.01560 & 0.00595 & 0.00043 & 0.00006 & 0.00003 & 0.02730 \\
\hline Total & 0.11165 & 0.37414 & 0.39648 & 0.09240 & 0.02528 & 0.00006 & 1.00000 \\
\hline
\end{tabular}


Table A.13 - Joint Occurrence Frequencies of Wind Direction Sector by Wind Speed Category for the 61m Level Central Climatology Tower, Winter 2004

\begin{tabular}{|c|c|c|c|c|c|c|c|}
\hline \multirow[b]{2}{*}{ Sector } & \multicolumn{6}{|c|}{ Wind Speed Category, meters/sec } & \multirow[b]{2}{*}{ Total } \\
\hline & $0-2$ & $2-4$ & $4-6$ & $6-8$ & $8-12$ & $>12$ & \\
\hline $\mathbf{N}$ & 0.00870 & 0.02530 & 0.02003 & 0.00458 & 0.00000 & 0.00000 & 0.05861 \\
\hline NNE & 0.00584 & 0.01866 & 0.02896 & 0.00870 & 0.00023 & 0.00000 & 0.06239 \\
\hline NE & 0.02141 & 0.01832 & 0.04052 & 0.00859 & 0.00240 & 0.00000 & 0.09123 \\
\hline ENE & 0.00412 & 0.01900 & 0.04682 & 0.01568 & 0.00229 & 0.00000 & 0.08791 \\
\hline E & 0.00675 & 0.01923 & 0.02621 & 0.00595 & 0.00011 & 0.00000 & 0.05826 \\
\hline ESE & 0.00469 & 0.01362 & 0.01877 & 0.00263 & 0.00000 & 0.00000 & 0.03972 \\
\hline SE & 0.00343 & 0.01202 & 0.01282 & 0.00069 & 0.00000 & 0.00000 & 0.02896 \\
\hline SSE & 0.00595 & 0.00996 & 0.01156 & 0.00080 & 0.00000 & 0.00000 & 0.02827 \\
\hline $\mathbf{S}$ & 0.00412 & 0.01202 & 0.01568 & 0.00687 & 0.00069 & 0.00000 & 0.03938 \\
\hline ssw & 0.00424 & 0.01007 & 0.02175 & 0.00401 & 0.00000 & 0.00000 & 0.04006 \\
\hline sw & 0.00572 & 0.01305 & 0.01969 & 0.00904 & 0.00103 & 0.00000 & 0.04853 \\
\hline WSW & 0.00538 & 0.02198 & 0.03423 & 0.02083 & 0.00229 & 0.00000 & 0.08471 \\
\hline WSW & 0.00424 & 0.02679 & 0.05666 & 0.04567 & 0.01351 & 0.00000 & 0.14686 \\
\hline WNW & 0.00641 & 0.01614 & 0.03743 & 0.01969 & 0.01053 & 0.00000 & 0.09020 \\
\hline NW & 0.00778 & 0.02324 & 0.01545 & 0.00195 & 0.00023 & 0.00000 & 0.04865 \\
\hline NNW & 0.00790 & 0.02839 & 0.00962 & 0.00034 & 0.00000 & 0.00000 & 0.04624 \\
\hline Total & 0.10668 & 0.28777 & 0.41621 & 0.15602 & 0.03331 & 0.00000 & 1.00000 \\
\hline
\end{tabular}

Table A.14 - Joint Occurrence Frequencies of Wind Direction Sector by Wind Speed Category for the 61m Level Central Climatology Tower, Spring 2004

\begin{tabular}{|c|c|c|c|c|c|c|c|}
\hline \multirow[b]{2}{*}{ Sector } & \multicolumn{6}{|c|}{ Wind Speed Category, meters/sec } & \multirow[b]{2}{*}{ Total } \\
\hline & $0-2$ & $2-4$ & 4-6 & $6-8$ & 8-12 & $>12$ & \\
\hline $\mathbf{N}$ & 0.00170 & 0.00951 & 0.01381 & 0.00136 & 0.00011 & 0.00000 & 0.02649 \\
\hline NNE & 0.00091 & 0.00566 & 0.00668 & 0.00215 & 0.00000 & 0.00000 & 0.01540 \\
\hline NE & 0.00170 & 0.00385 & 0.00589 & 0.00249 & 0.00000 & 0.00000 & 0.01393 \\
\hline ENE & 0.00181 & 0.00623 & 0.01019 & 0.00374 & 0.00000 & 0.00000 & 0.02197 \\
\hline E & 0.00260 & 0.01189 & 0.01902 & 0.00204 & 0.00000 & 0.00000 & 0.03555 \\
\hline ESE & 0.00589 & 0.01868 & 0.02785 & 0.00351 & 0.00011 & 0.00000 & 0.05605 \\
\hline SE & 0.00940 & 0.03363 & 0.03329 & 0.00079 & 0.00000 & 0.00000 & 0.07711 \\
\hline SSE & 0.01234 & 0.04065 & 0.06850 & 0.00147 & 0.00000 & 0.00000 & 0.12296 \\
\hline $\mathbf{s}$ & 0.00544 & 0.03702 & 0.06454 & 0.00510 & 0.00023 & 0.00000 & 0.11232 \\
\hline ssw & 0.00487 & 0.03080 & 0.04857 & 0.00544 & 0.00023 & 0.00000 & 0.08990 \\
\hline sw & 0.00566 & 0.03295 & 0.04337 & 0.00827 & 0.00226 & 0.00000 & 0.09250 \\
\hline wsw & 0.00600 & 0.03306 & 0.04461 & 0.01823 & 0.00555 & 0.00000 & 0.10745 \\
\hline wsw & 0.00317 & 0.02321 & 0.04291 & 0.02231 & 0.00781 & 0.00000 & 0.09941 \\
\hline WNW & 0.00283 & 0.01404 & 0.02706 & 0.01574 & 0.00883 & 0.00000 & 0.06850 \\
\hline NW & 0.00351 & 0.01449 & 0.01336 & 0.00770 & 0.00136 & 0.00000 & 0.04042 \\
\hline NNW & 0.00249 & 0.01042 & 0.00611 & 0.00068 & 0.00023 & 0.00011 & 0.02004 \\
\hline Total & 0.07031 & 0.32609 & 0.47577 & 0.10100 & 0.02672 & 0.00011 & 1.00000 \\
\hline
\end{tabular}


Table A.15 - Joint Occurrence Frequencies of Wind Direction Sector by Wind Speed Category for the $61 \mathrm{~m}$ Level Central Climatology Tower, Summer 2004

\begin{tabular}{|c|c|c|c|c|c|c|c|}
\hline \multirow[b]{2}{*}{ Sector } & \multicolumn{6}{|c|}{ Wind Speed Category, meters/sec } & \multirow[b]{2}{*}{ Total } \\
\hline & $0-2$ & 2-4 & 4-6 & 6-8 & 8-12 & $>12$ & \\
\hline $\mathbf{N}$ & 0.00567 & 0.01688 & 0.01179 & 0.00045 & 0.00000 & 0.00011 & 0.03490 \\
\hline NNE & 0.00510 & 0.01994 & 0.02085 & 0.00215 & 0.00023 & 0.00000 & 0.04827 \\
\hline NE & 0.00635 & 0.03581 & 0.02289 & 0.00193 & 0.00057 & 0.00000 & 0.06754 \\
\hline ENE & 0.00544 & 0.02697 & 0.01926 & 0.00079 & 0.00000 & 0.00000 & 0.05246 \\
\hline E & 0.00521 & 0.02720 & 0.01292 & 0.00136 & 0.00023 & 0.00000 & 0.04691 \\
\hline ESE & 0.00646 & 0.03003 & 0.01439 & 0.00283 & 0.00023 & 0.00000 & 0.05394 \\
\hline SE & 0.00748 & 0.02935 & 0.01462 & 0.00170 & 0.00011 & 0.00000 & 0.05326 \\
\hline SSE & 0.00895 & 0.02901 & 0.02006 & 0.00079 & 0.00011 & 0.00000 & 0.05892 \\
\hline$s$ & 0.00975 & 0.04249 & 0.02198 & 0.00057 & 0.00000 & 0.00000 & 0.07479 \\
\hline ssw & 0.00759 & 0.05099 & 0.02198 & 0.00079 & 0.00011 & 0.00000 & 0.08147 \\
\hline sw & 0.01065 & 0.05926 & 0.02776 & 0.00045 & 0.00034 & 0.00000 & 0.09847 \\
\hline wsw & 0.00963 & 0.06255 & 0.03626 & 0.00397 & 0.00000 & 0.00000 & 0.11241 \\
\hline wsw & 0.00907 & 0.05371 & 0.03773 & 0.00771 & 0.00034 & 0.00000 & 0.10856 \\
\hline WNW & 0.00827 & 0.02448 & 0.01564 & 0.00181 & 0.00045 & 0.00000 & 0.05065 \\
\hline NW & 0.00929 & 0.01575 & 0.00465 & 0.00057 & 0.00011 & 0.00000 & 0.03037 \\
\hline NNW & 0.00669 & 0.01439 & 0.00544 & 0.00057 & 0.00000 & 0.00000 & 0.02708 \\
\hline Total & 0.12159 & 0.53881 & 0.30822 & 0.02844 & 0.00283 & 0.00011 & 1.00000 \\
\hline
\end{tabular}

Table A.16 - Joint Occurrence Frequencies of Wind Direction Sector by Wind Speed Category for the $61 \mathrm{~m}$ Level Central Climatology Tower, Fall 2004

\begin{tabular}{|c|c|c|c|c|c|c|c|}
\hline \multirow[b]{2}{*}{ Sector } & \multicolumn{6}{|c|}{ Wind Speed Category, meters/sec } & \multirow[b]{2}{*}{ Total } \\
\hline & $0-2$ & $2-4$ & 4-6 & $6-8$ & 8-12 & $>12$ & \\
\hline $\mathbf{N}$ & 0.00527 & 0.01179 & 0.00561 & 0.00023 & 0.00000 & 0.00000 & 0.02289 \\
\hline NNE & 0.00618 & 0.02026 & 0.03846 & 0.00790 & 0.00046 & 0.00000 & 0.07326 \\
\hline NE & 0.00996 & 0.04842 & 0.10096 & 0.03205 & 0.00584 & 0.00000 & 0.19723 \\
\hline ENE & 0.03033 & 0.03800 & 0.04865 & 0.00893 & 0.00332 & 0.00000 & 0.12924 \\
\hline E & 0.00618 & 0.02232 & 0.02209 & 0.00206 & 0.00458 & 0.00000 & 0.05723 \\
\hline ESE & 0.00584 & 0.01889 & 0.00756 & 0.00263 & 0.00309 & 0.00000 & 0.03800 \\
\hline SE & 0.00687 & 0.01271 & 0.00778 & 0.00321 & 0.00435 & 0.00000 & 0.03491 \\
\hline SSE & 0.00813 & 0.01500 & 0.02232 & 0.00321 & 0.00355 & 0.00000 & 0.05220 \\
\hline s & 0.01202 & 0.02839 & 0.02324 & 0.00137 & 0.00195 & 0.00000 & 0.06696 \\
\hline ssw & 0.01088 & 0.02198 & 0.01671 & 0.00092 & 0.00126 & 0.00000 & 0.05174 \\
\hline sw & 0.01225 & 0.02358 & 0.01419 & 0.00446 & 0.00240 & 0.00000 & 0.05689 \\
\hline wsw & 0.01122 & 0.02518 & 0.02598 & 0.01328 & 0.00218 & 0.00000 & 0.07784 \\
\hline WSW & 0.00515 & 0.02370 & 0.01877 & 0.00710 & 0.00538 & 0.00000 & 0.06010 \\
\hline WNW & 0.00653 & 0.01179 & 0.01248 & 0.00160 & 0.00103 & 0.00000 & 0.03343 \\
\hline NW & 0.00813 & 0.01316 & 0.00343 & 0.00046 & 0.00000 & 0.00000 & 0.02518 \\
\hline NNW & 0.00572 & 0.01362 & 0.00343 & 0.00011 & 0.00000 & 0.00000 & 0.02289 \\
\hline Total & 0.15064 & 0.34879 & 0.37168 & 0.08951 & 0.03938 & 0.00000 & 1.00000 \\
\hline
\end{tabular}

